\title{
Application of Lattice Boltzmann and Navier-Stokes Methods to NASA's Wall Mounted Hump
}

\author{
Gerrit-Daniel Stich, Jeffrey A. Housman’ \\ Joseph G. Kocheemoolayil $\$$ Michael F. Barad $\$$, and Cetin C. Kiris, \\ NASA Ames Research Center, M/S 258-2, Moffett Field, CA 94035
}

\begin{abstract}
Lattice Boltzmann (LB) based Large Eddy Simulation (LES), Reynolds-averaged NavierStokes (RANS) as well as hybrid RANS/LES methods within the Launch Ascent and Vehicle Aerodynamics (LAVA) solver framework are applied to NASA's wall-mounted hump. Computational results are compared with experiments performed by Greenblatt et al. ${ }^{1}$ A detailed comparison between the accuracy and resolution requirements of the two approaches for turbulence resolving simulations, as well as the suitability of different grid paradigms (body-fitted curvilinear and block structured Cartesian) are presented. This test case is part of NASA's Revolutionary Computational Aerosciences (RCA) sub-project which addresses the technical challenge of predicting flow separation and reattachment accurately. Improvements in predictive accuracy by as much as $\mathbf{9 0 \%}$ are demonstrated using LB as well as hybrid RANS/LES approaches compared to state-of-the-art steady state RANS simulations.
\end{abstract}

\section{Introduction}

The NASA Revolutionary Computational Aerosciences (RCA) sub-project has created a technical challenge to identify and down-select critical turbulence, transition, and numerical method technologies for $40 \%$ reduction in predictive error. The test cases within the RCA technical challenge include turbulent separated flows, evolution of free shear flows and shock-boundary layer interactions on canonical configurations. As current turbulence models within state-of-the-art RANS solvers struggle to predict the shape and size of separation and reattachment regions, the focus has shifted towards eddy resolving approaches. In an effort to address some of those technical challenges, Lattice Boltzmann (LB), as well as hybrid Reynoldsaveraged Navier-Stokes/large-eddy simulation (RANS/LES) methods within the Launch Ascent and Vehicle Aerodynamics (LAVA) solver framework are applied to the 2D NASA wall-mounted hump test case from Greenblatt et al. ${ }^{1}$ This test case has been previously studied using both numerical approaches, the classical Navier-Stokes $(\mathrm{NS})^{2,3}$ as well as the emerging Lattice Boltzmann method (LBM), ${ }^{4}$ but no detailed comparison of the advantages and disadvantages between the two approaches regarding accuracy and resolution requirements has been performed. This motivated the current study.

This paper is organized as follows: Sections II \& III describe the test case and the underlying numerical method used. First, the SA model implementation within the body-fitted curvilinear overset grid methodology is assessed and the challenges RANS models face in predicting smooth body flow separation are outlined. Second, in Section IV.B delayed detached eddy (DDES) simulation results as well as zonal detached eddy (ZDES) results are presented and compared. The issues arising from the gray area problem ${ }^{5}$ resulting in a delayed transition towards 3D turbulent structures in the separated shear-layer are presented. Finally computational results obtained with the LBM-LES method are presented in Section IV.C and compared with the hybrid RANS/LES simulations as well as results from literature ${ }^{3,4}$ and measurements. ${ }^{1}$ A fair

\footnotetext{
*Science and Technology Corporation, AIAA Member, gerrit-daniel.stich@nasa.gov

${ }^{\dagger}$ Computational Aerosciences Branch, AIAA Senior Member, jeffrey.a.housman@nasa.gov

${ }^{\ddagger}$ Science and Technology Corporation, AIAA Member, joseph.g.kocheemoolayil@nasa.gov

$\S$ Computational Aerosciences Branch, AIAA Senior Member, michael.f.barad@nasa.gov

๑ Computational Aerosciences Branch, AIAA Senior Member, cetin.c.kiris@nasa.gov
} 
comparison between the different methods considering accuracy and resolution requirements is presented in the summary (Sec. V).

\section{Test Case - NASA Wall-Mounted Hump}

The test case ${ }^{1}$ studied in this paper is based on the experimental database of the ERCOFTAC a framework and describes a low-speed flow separation over a wall-mounted hump geometry. The setup of the GlauertGoldschmied type body can be seen in Fig. 1 and is similar to the setup of Seifert et al. ${ }^{6}$ It is mounted on a splitter plate at the wind tunnel floor between two side plates $0.584 \mathrm{~m}$ apart from each other and has a chord length of $c=0.42 \mathrm{~m}$.

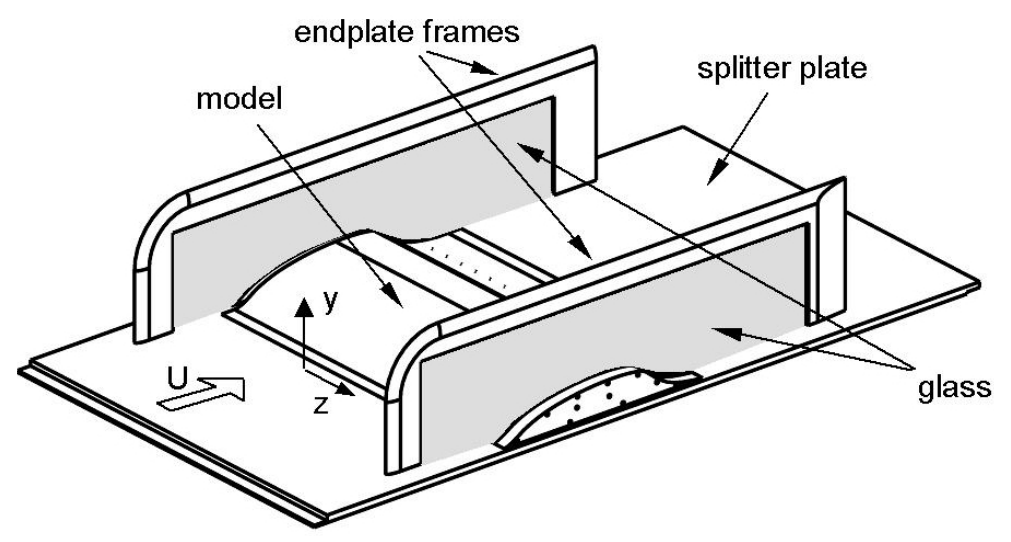

Figure 1: Experimental setup of NASA wall-mounted hump by Greenblatt et $a l^{1}$

The side-plates shown in the experiments are neglected in the simulations and periodic boundary conditions are applied in the spanwise direction with a width of 0.2c. Simulations with the same lateral extent have been previously carried out by You et al. ${ }^{7}$ and Uzun and Malik ${ }^{3}$ and good agreement with skin friction and pressure coefficient distrubution has been achieved. As recommended by the NASA CFDVAL2004 workshop a contoured top wall was used in the simulation to account for the side-wall blockage effects and is located at $\mathrm{y} / \mathrm{c} \approx 0.9 \mathrm{c}$. A study on the effect of this top wall contour can be found in Uzun and Malik ${ }^{8}$ who also point out limitations of this simplified approach. The reported Reynolds number based on freestream velocity and chord length is $R e_{c}=9.36 \times 10^{5}$ with a Mach number of $M a_{\infty}=0.1$ and a momentum thickness Reynolds number of $R e_{\Theta}=7200$. It should be noted that experiments and extensive skin friction measurements for the same configuration and flow condition have been performed by Naughton et al., however a slightly lower momentum thickness Reynolds number value of 6800 has been reported. The small slot at the leeward side of the hump located at $x=0.65 c$, which was implemented for flow control purposes, is not modeled as its effect was negligible for the mean statistics obtained in both experiments and previous simulations.

\section{Computational Methodology}

The LAVA solver framework ${ }^{9}$ is utilized for the computational study. LAVA offers flexible meshing options and was developed with the intent of modeling highly complex geometry and flow-fields. The framework supports Cartesian and curvilinear structured grids as well as unstructured arbitrary polyhedral meshes. Overset grid technology ${ }^{10}$ is used to couple the solutions across different overlapping meshes. In this study, the Cartesian grid approach is used with the LBM-LES method and the structured curvilinear overlapping grid methodology is used for the RANS and hybrid RANS/LES method. The Cartesian grids are automatically generated within the LAVA framework and the Pointwise ${ }^{\mathrm{TM}}$ and Chimera Grid Tools $(\mathrm{CGT})^{11}$ software packages are used to generate the curvilinear grids.

\footnotetext{
${ }^{\mathrm{a}}$ ERCOFTAC: European Research Community On Flow, Turbulence And Combustion
} 


\section{III.A. Curvilinear Navier-Stokes Solver}

The compressible hybrid Reynolds Averaged Navier-Stokes/Large Eddy Simulation (RANS/LES) equations are solved using a finite-difference formulation applied to the curvilinear transformed system of equations in strong conservation law form. ${ }^{12}$ The Spalart-Allmaras (SA) ${ }^{13}$ turbulence model is used as the base RANS closure model. Two hybrid RANS/LES modeling approaches are assessed in this paper, including a zonal and a non-zonal formulation. Implicit second-order backward differencing (BDF2) is used for time integration and the discretized equations are marched in pseudo-time until a sufficient reduction in the residual has been achieved for each physical time-step (approximately 3 to 4 orders of magnitude of residual reduction is achieved in the present computations). The nonlinear system of equations are linearized at each pseudo timestep and an alternating line-Jacobi relaxation procedure is applied. A local pseudo time-step corresponding to a Courant-Friedrichs-Lewy (CFL) of 10 is used to accelerate convergence. Domain decomposition and the Message Passing Interface (MPI) are used to enable a scalable parallel algorithm.

\section{III.A.1. Low Dissipation Finite-Difference Method}

High-order accurate low dissipation finite-difference schemes have been shown to be an effective strategy for turbulence resolving simulations using LAVA. ${ }^{14-17}$ A thorough study comparing several high-order finitedifference methods on Cartesian grids within the LAVA framework was reported previously. ${ }^{18}$ Results from this study indicated that high-order Weighted Essentially Non-Oscillatory (WENO) schemes ${ }^{19}$ performed well in both resolution (Points-Per-Wavelength PPW), shock capturing, and robustness under harsh flow conditions. A natural extension of finite-difference WENO schemes to curvilinear grids are the high-order Weighted Compact Nonlinear Schemes (WCNS) ${ }^{20}$ The WCNS method, applied to the convective fluxes, consists of WENO interpolation (as opposed to reconstruction) of the left and right states to the half grid points, followed by evaluation of the numerical flux at the half points by an approximate (or exact) Riemann solver or flux vector splitting scheme, and concluding with a high-order central finite-difference operator at the grid points which depends on the numerical fluxes at the half points in either an implicit (i.e. compact) or explicit form. When applying finite-difference methods to the curvilinear equations in strong conservation law form, standard WENO finite-difference methods will not satisfy the Geometric Conservation Law (GCL) making it necessary to combine the WENO interpolation with high-order central-difference operators. It has been shown that free-stream preservation (i.e. the GCL condition) is satisfied up to machine precision provided that identical central difference operators are used for discretizing the metric terms as well as the fluxes. ${ }^{21,22}$ An additional advantage of WCNS over WENO is the ability to use approximate Riemann solvers. Standard finite-difference WENO methods require the use of flux vector splitting methods for numerical flux evaluation. In this work, a modified version of the Roe numerical flux is used. ${ }^{23-25}$

A consequence of using high-order central difference operators applied to numerical fluxes at the half grid points, which depend on high-order WENO interpolation, is the much wider stencil required for the same order of accuracy compared to the standard finite-difference WENO method. To reduce this pathology, high-order central difference operators using a combination of the numerical fluxes at the half grid points and the physical fluxes at the grid points have been developed. ${ }^{26,27}$ This approach, denoted Hybrid Weighted Compact Nonlinear Scheme (HWCNS), allows for up to third/fourth-order accuracy using a five-point stencil by combining blended third- and fourth-order interpolation with a fourth-order hybrid central difference operator. In the current approach, the convective fluxes (and the metric-terms used within) are discretized with the high-order HWCNS, while the viscous fluxes (and their metric terms) are discretized with standard second-order accurate central differencing. A more detailed description is included in Housman et al. ${ }^{16}$

\section{III.A.2. Non-Zonal Hybrid RANS/LES model}

The Detached Eddy Simulation ${ }^{5,28}$ (DES) and Delayed Detached Eddy Simulation ${ }^{29-31}$ (DDES) turbulence model closures are well-tested hybrid RANS/LES models for highly separated flows. In the original DES model, the transition between RANS and LES models was based strictly on local mesh size relative to the wall-distance. For geometries with a wide range of geometric length scales, such as a high-lift devices with finite-thickness leading and trailing edges or nozzles with finite thickness exits, the local mesh spacing may become small enough to force transition from the RANS model to the LES model, but the mesh is typically not small enough to resolve the unsteady fluctuations causing the well-known modeled stress depletion. ${ }^{32}$ This resulted in a modification denoted as DDES, which attempts to remain in RANS mode in the attached boundary layer. ${ }^{29}$ Inspection of the shielding function often shows a strange behavior of going from RANS 
near the wall, to LES, back to RANS just past the edge of the boundary layer, and subsequently back to LES. ${ }^{33}$

One critique of hybrid RANS/LES models is the slow development of three-dimensional turbulent structures even when the spatial and temporal resolution are adequate to capture them. This is often caused by the definition of the local length scale in the model, which for the DDES model is the largest edge length associated with the cell (or dual cell) of a grid point. An alternative length scale definition developed using the Zonal Detached Eddy Simulation (ZDES) approach ${ }^{34,35}$ utilizes a normalized vorticity vector together with combinations of two-dimensional length scale estimates. The normalized vorticity vector allows the span-wise or azimuthal direction to be identified in the initial onset of two-dimensional instabilities, and removes the grid length spacing in that direction (which may be large compared to the streamwise and shear directions) from the local length scale estimate. This reduction is effectively ignored once three-dimensional turbulent structures are formed. At this point the normalized vorticity vector will not be aligned with any particular mesh direction and the standard length scale is recovered. Utilization of the normalized vorticity vector along with a more conservative estimate of the mesh spacing has been developed ${ }^{36}$ and is used in the present work. A detailed description of the length scale definition is included in Housman et al. ${ }^{16}$

\section{III.A.3. Zonal Hybrid RANS/LES model}

An alternative strategy appropriate for structured multi-block and overset grids is the Zonal DES (ZDES) approach first introduced by Deck ${ }^{35,37,38}$ in which specific zones are designated to use the RANS, DDES, or LES models explicitly. This idea of zonal specification has been further generalized to include a user-specified wall distance based transition location between RANS and LES. ${ }^{39,40}$ This allows the user to choose, based on a strong understanding of the physics of the problem, which regions should be solved in pure RANS and hybrid RANS/LES mode. When in hybrid RANS/LES mode it also gives additional control to the user to prescribe the transition location to explicitly guarantee that the attached boundary layer remains in RANS mode. This is very important since shielding functions, such as those used in the DDES model, can still fail when the mesh is fine enough to capture some three-dimensional fluctuations, but not fine enough to resolve the relevant scales in the boundary layer to accurately predict skin friction.

A recent extension to ZDES, introduced by Deck et al. ${ }^{35,41,42}$ and improved by Renard et al. ${ }^{43}$ in which the model acts in a wall-modeled LES (WMLES) mode has also been added to LAVA. Here RANS is used in the inner layer of the attached boundary layer up to a user selected wall distance (typically $10 \%$ of local BL thickness ${ }^{43}$ ), and interfaces to an outer LES (no forcing or filtering is applied). This is a simple and robust approach which avoids spurious artifacts at the interface, however it can lead to a log-layer mismatch caused by the inconsistency in the equation set across the interface (i.e. Reynolds averaged Navier-Stokes on one side of the interface and filtered Navier-Stokes on the other side).

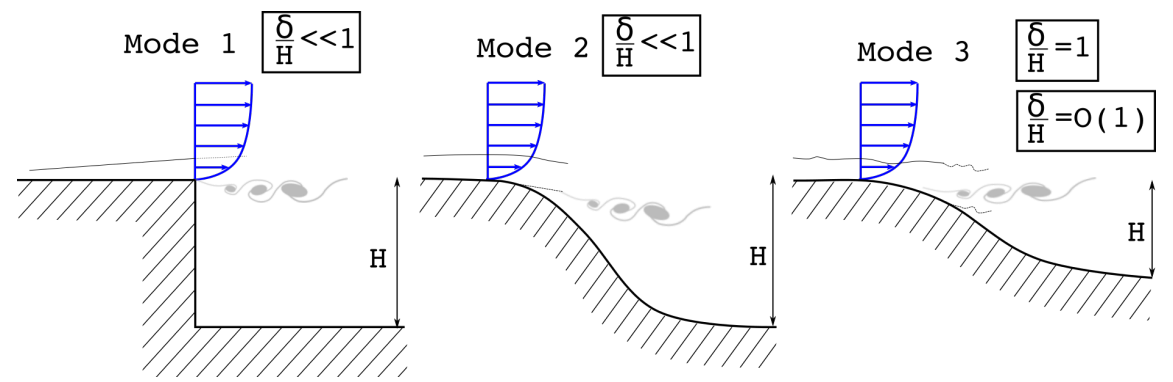

Figure 2: Classification of canonical flow problems by mode following Deck et al.: ${ }^{35}$ (1) separation fixed by geometry, (2) separation induced by pressure gradient on curved surface, (3) separation strongly dependent on dynamics of incoming boundary layer.

The ZDES approach used in this paper is based on the Spalart-Allmaras (SA) turbulence model, where the pseudo viscosity $\widetilde{\nu}_{t}$ from the model scales with the distance to the wall $d_{w}$ and the local vorticity magnitude $\Omega$ in the inner layer. In the outer LES region the SA model acts as a subgrid-scale model (SGS) with a modified length scale for $d_{w}$. For this reason Deck introduced three different hybrid length scale formulations replacing $d_{w}$ with $d_{\text {ZDES }}$ which were optimized for canonical flow configurations (see Figure 2). Only Mode 3 , which is calibrated for flows where the separation is strongly influenced by the dynamics of the incoming boundary layer, will be considered in this study. The user must select which zones to solve in pure RANS 
mode and which zones to apply ZDES Modes 1, 2, or 3. For zones which use ZDES Mode 3, the user must also prescribe an interface distance from the wall $h_{w}$ at which to transition from RANS to LES. A detailed discussion about the interface location can be found in Section IV.B.1. The user must also suppy turbulent fluctuations at the inflow (Sec. III.C). The hybrid length scale for Mode 3 is defined in the following way:

$$
d_{\mathrm{ZDES}}= \begin{cases}d_{w}, & \text { if } d_{w}<h_{w} \\ \min \left(d_{w}, C_{\mathrm{DES}} \cdot \Delta\right), & \text { otherwise }\end{cases}
$$

with the constant $C_{\mathrm{DES}}=0.65$ and the subgrid length scale $\Delta=(\Delta x \Delta y \Delta z)^{1 / 3}$.

\section{III.B. Cartesian Lattice Boltzmann Solver}

Over the past two decades, the Lattice Boltzmann Method has matured into a burgeoning technique for simulating engineering fluid flows of practical importance. The LB method is a mesoscopic approach wherein simplified kinetic equations that retain just enough detail to satisfy the desired macroscopic equations of fluid motion (weakly compressible, isothermal Navier-Stokes equations in the present context) are solved. ${ }^{44}$ The local state of fluid motion is described by density distribution functions $f(\vec{x}, t, v)$, which upon being normalized by the local density represent the probability of finding particles moving with velocity $v$ in an infinitesimal volume $d x$ about $\vec{x}$. The familiar macroscopic variables such as density and the components of momentum are determined from the density distribution functions through moment summations. Details of the implementation of the LB method and recent improvements within the LAVA framework can be found in Barad et al. ${ }^{45,46}$

\section{III.B.1. Governing Equations}

The LB equation governs the space-time evolution of density distribution functions $f(\vec{x}, t, v)$. The density distribution function $f(\vec{x}, t, v)$ at a particular node of the lattice defines the fraction of mass contained in a control volume surrounding the node which moves at velocity $v$. For example, $f(\vec{x}, t, 0)$ defines the fraction of mass contained in the control volume which is at rest. The equation is solved numerically through an extremely efficient collide at nodes and stream along links algorithm. In a single time step, the virtual computational particles collide at a node relaxing towards the local equilibrium and subsequently hop on to the neighboring nodes of the lattice. The velocity space is commonly discretized into 15, 19 or 27 velocities on a regular cubic lattice in $3 \mathrm{D}$, where the notation $D d Q q$ describes a lattice in $d$ dimensions with $q$ discrete velocities. The local equilibrium is a truncated, low-speed approximation of the Maxwell-Boltzmann distribution function corresponding to the local macroscopic variables.

The collision operation is perfectly local in space and time. Every node performs this operation independent of every other node in the lattice. It is perfectly non-local in the velocity space within a given cell in the physical space, i.e. all particles interact with each other during the collision process. The velocity space is discrete. The particles are only allowed to have a certain number of discrete velocities consistent with discrete node-to-node streaming operations on the lattice. The particles are bound to the lattice, and are not allowed to occupy positions in between the nodes of the lattice. They simply hop from one node to another on the lattice as time advances. This makes exact, dissipation-free advection (i.e. streaming) a simple nearest-neighbor 'copy' operation.

The fluid viscosity sets the rate at which the density distribution functions relax to the local equilibrium. More viscosity implies faster relaxation to the local equilibrium and lower deviations from the local equilibrium on average. The relaxation rate is also inversely related to the local lattice spacing. A coarser mesh results in larger deviations from the local equilibrium for a given viscosity. For small Mach numbers, the Chapman-Enskog expansion can be used to show that the simplified collision rules with truncated equilibrium distribution functions reproduce the weakly-compressible, isothermal Navier-Stokes equations in the low-frequency/large-wavelength limit. ${ }^{47}$

\section{III.B.2. Collision Models}

The following collision models are currently available in the solver:

1. Bhatnagar-Gross-Krook (BGK) model - All distribution functions relax to their local equilibrium values at the same constant rate for every node in a given grid refinement level. ${ }^{48}$ All moments of the 
distribution function (from order two to six) relax to their equilibrium values at a single rate that is only a function of the fluid viscosity and the local lattice spacing.

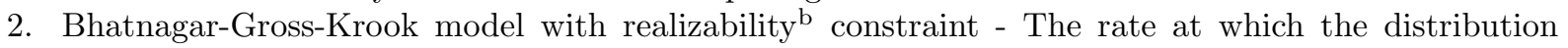
functions relax to their local equilibrium is modified locally at every node of the lattice to enforce strict realizability of the distribution functions everywhere. ${ }^{49}$ Strict enforcement of realizability through local under-relaxation (if and where necessary) is tantamount to surgical addition of artificial viscosity in regions where the simulated flow is too under-resolved to maintain numerical stability. This approach guarantees nonlinear stability and makes engineering Reynolds numbers accessible at a manageable computational cost.

3. Entropic model - The rate at which the distribution functions relax to their local equilibrium is modified locally to enforce compliance with the H-theorem. ${ }^{50}$ The local relaxation rate is modified to ensure that the Boltzmann-Shannon entropy of the post collision state is not lower than the pre-collision state. ${ }^{50}$ Since the Boltzmann-Shannon entropy is a Lyapunov function in the phase space corresponding to the density distribution functions, compliance with H-theorem guarantees nonlinear stability. Compliance with H-theorem also guarantees realizability by construction.

4. Multiple-Relaxation Time (MRT) model - The collision operation is performed in the moment space. Conceptually, the pre-collision density distribution functions are transformed into the moment space through a linear transformation and post-collision moments transformed back into the velocity space. The higherorder moments (the so-called ghost modes) are relaxed to equilibrium at a faster rate relative to the hydrodynamic modes. ${ }^{51}$

5. Entropic Multiple-Relaxation Time (EMRT) model - Instead of choosing the relaxation rates for the higher-order moments in an ad hoc manner for the entire lattice, they are chosen locally on a node-to-node basis based on the entropic principle. ${ }^{52}$ All the LB simulations presented in this paper were performed using the entropic multi-relaxation time model on the D3Q27 lattice.

6. Regularized Bhatnagar-Gross-Krook model - The pre-collision state is regularized by equilibrating the higher-order moments using Grad's approximation. ${ }^{53}$ The post-collision state is obtained from the regularized pre-collision state using the standard Bhatnagar-Gross-Krook (BGK) rule.

The unresolved, subgrid scales of turbulence are modeled using the constant-coefficient Smagorinsky model,${ }^{54}$ which modifies the local rate at which the distribution functions relax to equilibrium.

\section{III.B.3. Boundary Conditions}

The LBM formulation accounts for the presence of an embedded geometry through simple, intuitive rules which express the unknown, incoming populations in terms of the known, outgoing populations in order to complete the streaming step for lattice links intercepted by the geometry. For example, simply bouncing the particles back from the geometry in the opposite direction enforces the no-slip boundary condition. Specular reflection of particles in the mirror image direction is realized as the free-slip/no-penetration boundary condition. Both of these standard bounce-back (SBB) rules approximate the actual curved geometry with a series of small steps whose sizes are proportional to the local grid spacing.

Interpolated bounce-back rules capture the curvature in geometry more accurately, albeit at the cost of forfeiting strict realizability and precise mass conservation. Our experiments indicate that the linear bounce-back (LBB) formulation proposed by Bouzidi et al. ${ }^{55}$ is accurate, sufficiently robust, and extremely efficient computationally. The LBB computational time penalty relative to the SBB rule is minimal for the Structure-of-Arrays (SoA) data structure we have employed in our solver, where the boundary condition routine can take advantage of excellent cache reuse.

A numerical sponge, where the relaxation time is ramped up, was used in a buffer zone close to far-field boundary to minimize spurious reflections. The treatment of complex geometries within the Cartesian solver framework is described in Barad et al. ${ }^{45}$

\section{III.B.4. Wall-modelling}

One of the major issues faced by LES simulations is handling high Reynolds numbers in wall bounded flows. This is exacerbated in Cartesian solvers with isotropic cells. In an attempt to alleviate those harsh requirements, models have been developed that mimic near-wall physics close to the wall. An overview of recent development and future directions on wall-modeled LES can be found in Larsson et al. ${ }^{56}$ and Bose

\footnotetext{
${ }^{\mathrm{b}} \mathrm{A}$ realizable post-collision state is one in which all relevant probability density functions take values between zero and one.
} 
and Park. ${ }^{57}$ The wall-model implemented in our framework solves Turbulent Boundary Layer equations within the first cell and replaces the Lattice Boltzmann density distribution functions near the wall.

The main difference between wall-models for Navier-Stokes LES and LBM-LES is that LBM unknowns near the wall are density distribution functions and not velocities. More details of the implemented wallmodel can be found in Malaspinas et al. ${ }^{58}$ We have extended the model to handle curved geometry. Details of this modification will be reported in a separate publication, along with an extensive verification and validation study.

\section{III.B.5. Multi-Resolution Cartesian Methods}

The Cartesian structured adaptive mesh refinement (SAMR) methodology is capable of automatically generating, refining, and coarsening nested Cartesian volumes given a closed surface triangulation, and hence, offers the ability to dynamically track important flow features as they develop. Figure 3a shows an idealized SAMR hierarchy refining about a feature. In adaptive methods, one adjusts the computational effort locally to target a uniform level of accuracy throughout the problem domain. Cartesian SAMR is a proven methodology for multi-scale problems, with an extensive existing mathematical and software knowledge base. ${ }^{59-64}$ The LAVA code incorporates data structures and inter-level SAMR operators from the high-performance Chombo library. ${ }^{65}$ SAMR allows the simulation of a wide range of spatial and temporal scales through local refinement.

The advancement of time with a locally refined Cartesian mesh hierarchy is typically either composite or sub-cycled. For the composite approach, a constraint is imposed that all SAMR levels are advanced with the same time step, i.e. $\Delta t=$ constant. The composite approach is not possible for the LB formulations considered here. The sub-cycling of levels is performed, as described in, ${ }^{61,66}$ where each level is advanced in such a way as to maintain a constant CFL, i.e. $\Delta t=C \Delta x$.

Figure $3 \mathrm{~b}$ illustrates the flow of information during the subcycling algorithm for a mesh with 3 levels. The right pointing red arrows indicate space-time interpolation from a coarse level to a fine level, filling ghost cells. The left arrows indicate an averaging down procedure from fine to coarse levels. The blue up arrows indicate a single level advancement (streaming step) in time. The coarse-fine algorithm ensures discrete conservation of mass and momentum to machine precision. ${ }^{67,68}$ Figure 3 a shows the algorithm for the left-to-right component of the density distribution function depicting streaming (blue), communication exchange from components streaming into a finer level (red) and from fine-to-coarse for outgoing density distribution functions (green). More information on this recursive algorithm can be found in Chen et al. ${ }^{67}$ and Rohde et al. ${ }^{68}$

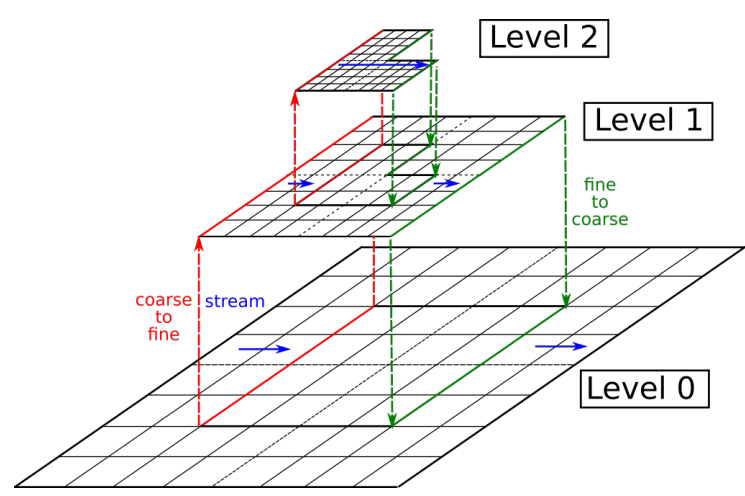

(a)

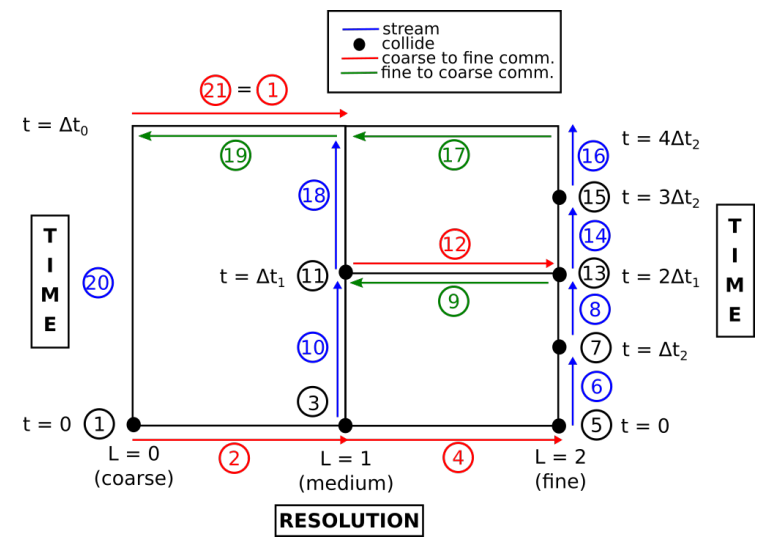

(b)

Figure 3: (a) Block structured adaptive mesh refinement showing 3 levels refined by factors of 2. Subcycle procedure demonstrated on left-to-right component of density distribution function: streaming (blue), coarseto-fine communicaton (red), fine-to-coarse communication (green). (b) Recursive sub-cycling algorithm for a 3 level hierarchy of grids. Arrows indicate direction of information propagation. 


\section{III.C. Turbulent Inflow Generation - Synthetic Eddy Method}

The synthetic eddy method (SEM) of Jarrin et. $a l^{69,70}$ is used to generate turbulent structures in the upstream boundary layer. This approach introduces synthetic eddies at the interface, such that first and second order turbulent statistics are matched with the upstream RANS solution. The eddies are convected at a characteristic velocity in order to mimic the temporal and spatial correlations of actual three-dimensional turbulence. Specification of the eddies and their downstream evolution are illustrated for a turbulent channel flow in Figure 4a. Once the governing equations are integrated forward in time for a sufficiently long duration, the turbulent statistics can be computed. Figure $4 \mathrm{~b}-\mathrm{d}$ show the time-averaged stream-wise velocity, the RMS stream-wise velocity, and the resolved turbulent kinetic energy. Although the interface will converge to the correct first and second order statistics, there is a delay before physical three-dimensional turbulence is recovered within the flow field. This can be observed in the RMS of the stream-wise velocity. Some modifications of the formulation have been made regarding the size of the eddies. ${ }^{71}$ Whenever used, the SEM interface is placed 3.0 chords upstream of the hump, which corresponds to more than 30 boundary layer thickness. The correlation length scales used in this implementation are computed (see Eqn. 2) using Bradshaws hypothesis to compute the turbulent kinetic energy.

$$
\sigma_{S E M}=\max \left(\min \left(2 d_{w}, 3 \frac{k^{2.5}}{\epsilon}\right), 1.5 \Delta\right)
$$

Where $\Delta=\max \left(\Delta_{x}, \Delta_{y}, \Delta_{z}\right), d_{w}$ the wall distance and $\epsilon=0.09 k^{2} / \nu_{t}$ the rate of dissipation. The turbulent kinetic energy $k$ is computed with $k=\frac{\nu_{t}|S|}{c_{\mu}}$.

\section{Computational Results}

Steady-state RANS as well as high-fidelity time-accurate simulations utilizing LBM-LES and NS-LES methods were performed using the LAVA solver framework. Multiple grid paradigms, namely structured curvilinear overset and immersed boundary Cartesian methods, were utilized and the success and challenges inherent to each method will be discussed and presented in this section. First, verification of the SA model implementation within the body-fitted curvilinear overset grid methodology is assessed by comparing to results posted on NASA's Turbulence Modeling Resource (TMR) website ${ }^{72}$ (Sec. IV.A). This configuration has proven to be a challenging test case for most RANS solvers in accurately predicting the bubble size and reattachment location. In Section IV.B two different approaches to hybrid RANS/LES (DDES and ZDES Mode 3) are compared. ZDES Mode 3 can also be considered a wall-modeled LES approach with RANS serving as the wall-model. In addition, the ability of the SEM method to force a more rapid transition towards three-dimensional structures in the shear layer that originates from the smooth body flow separation is investigated. The sensitivity of the results to the interface location as well as the time step in the ZDES Mode 3 simulations are presented in Sec. IV.B.1 and IV.B.2 respectively. Finally in Sec. IV.C the LatticeBoltzmann method in LES mode is applied and results are compared with the ZDES results and with existing wall-resolved ${ }^{38}$ and wall-modeled ${ }^{73}$ simulations from the literature. We conclude with a unbiased comparison between ZDES Mode 3 and LBM-LES.

\section{IV.A. Validation and Challenges of Reynolds-Averaged Navier-Stokes Simulations}

In this section results from 2D RANS simulations on three different curvilinear meshes are presented and the challenges this method faces are highlighted. The three finest grids from the $\mathrm{TMR}^{74}$ website without plenum have been used for this study. Previous works have shown that it is very important to resolve the shear layer as well as the separation and re-attachment location adequately to predict the size of the separation bubble. Hence clustering downstream of the separation point, the re-attachment point and in the favorable pressure gradient region upstream of the hump is applied. Figure 5 shows a succession of the three grids used. The corresponding grid sizes for the entire domain are coarse (205x55), medium (409x109) and fine (817x217). In order to run LAVA on these grids, the $2 \mathrm{D}$ slices where copied to three planes in order to create a 3D grid.

The steady-state RANS calculations are performed using a 2nd order modified Roe scheme without limiting or preconditioning. The Spallart-Allmaras $(\mathrm{SA})^{13}$ turbulence model without the so called "ft2" term was utilized in this work. The SA working variable $\tilde{\nu}_{t}$ is set to $3 \nu_{\infty}$ as recommended for the SA-noft2 turbulence model. ${ }^{72}$ The simulations were run with a constant CFL number until the $L_{2}$ norm of the flow residual on each zone was reduced by at least five orders of magnitude from its initial value. 


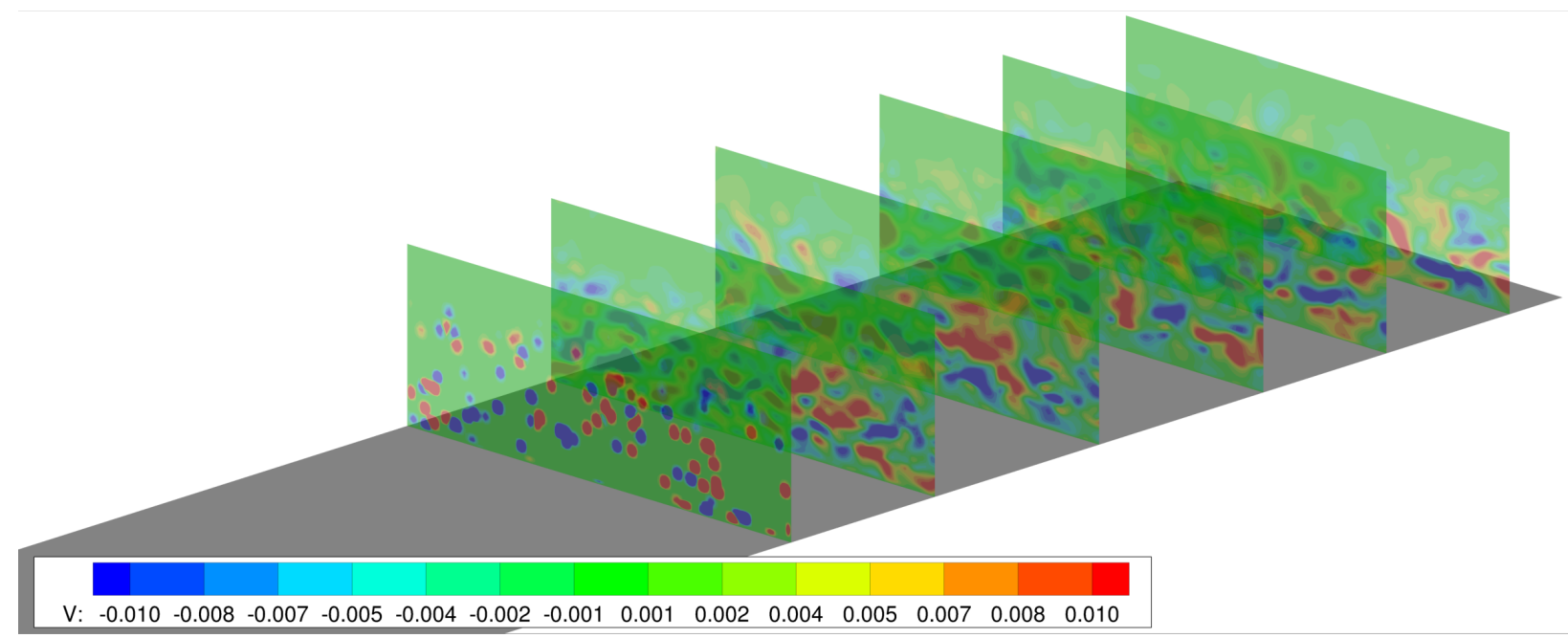

(a) Instantaneous span-wise velocity

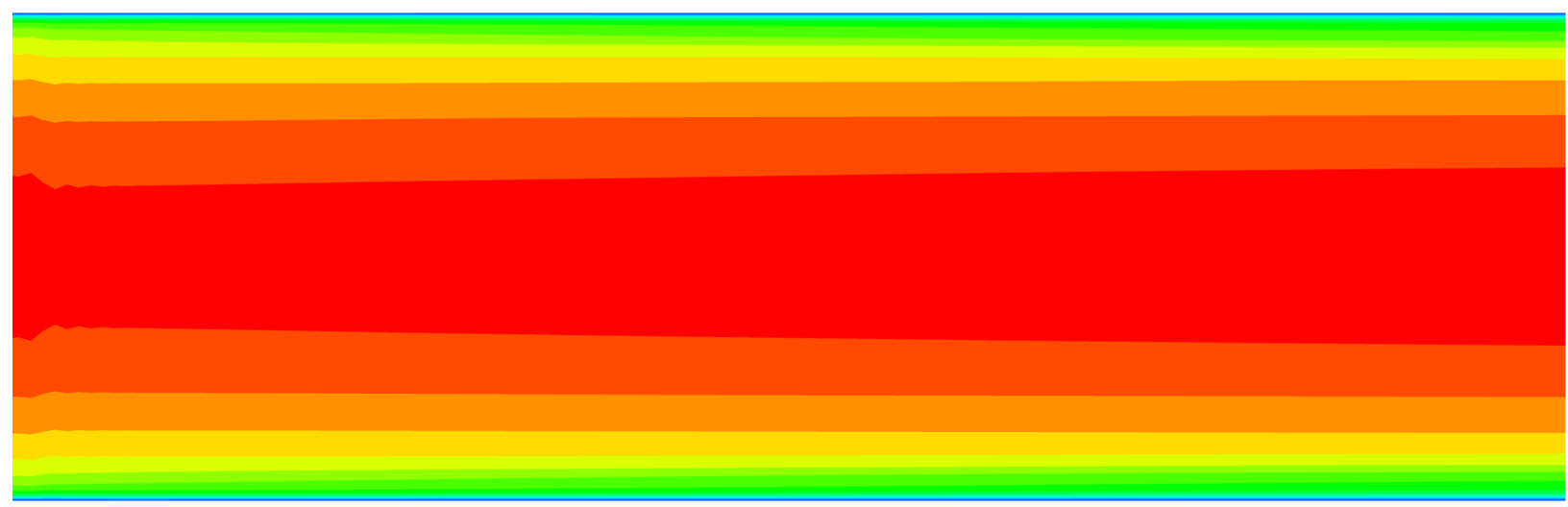

Uavg: $0.00 \quad 0.020 .040 .06 \quad 0.08 \quad 0.100 .120 .14 \quad 0.16 \quad 0.18 \quad 0.20 \quad 0.22 \quad 0.24 \quad 0.26$

(b) Time-averaged stream-wise velocity

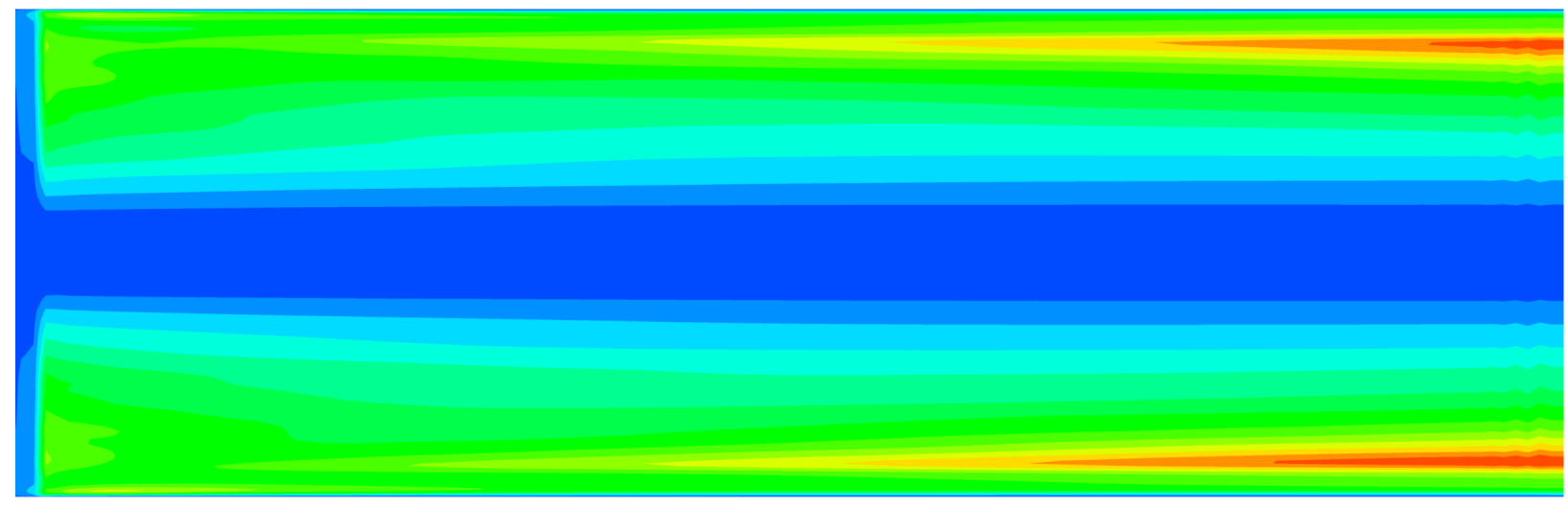

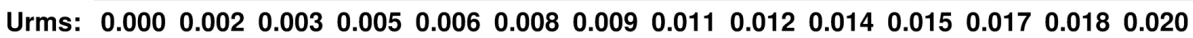

(c) RMS stream-wise velocity

Figure 4: (a) Isometric view of the instantaneous span-wise velocity illustrating the synthetic eddies inserted into the channel flow at the first plane, and their evolution downstream. (b) Time-averaged stream-wise velocity showing a small perturbation in the boundary layer where the synthetic eddies are inserted and the recovery of the boundary layer downstream. (c) RMS stream-wise velocity showing the development of the resolved turbulent content downstream of the SEM interface. 

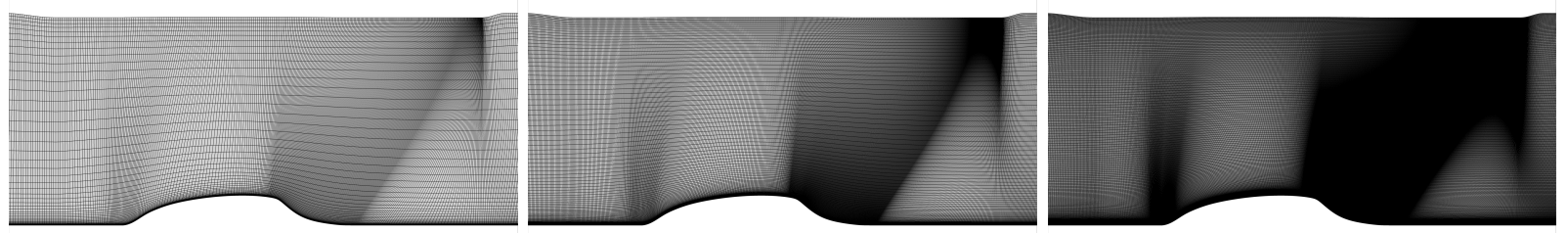

Figure 5: Coarse $(205 \times 55)$, medium (409x109) and fine (817x217) RANS mesh from TMR. ${ }^{74}$

As this case is part of the NASA RCA Technical Challenge, defined in NASA's CFD Vision 2030 Study, ${ }^{75}$ specific validation metrics have been defined. Those are the location of separation and reattachment, the turbulent shear stress and velocity profiles as well as skin friction and surface pressure coefficients. Of interest is also the deviation of the separation bubble size (Eqn. 3) and the variance in the peak turbulent shear stress magnitude (Eqn. 4).

$$
\begin{gathered}
\delta_{\text {bubble }}=\left[\left((x / c)_{\text {rea }}-(x / c)_{\text {sep }}\right)-0.435\right] / 0.435 \\
\delta_{u^{\prime} v^{\prime}}=-\left[\left(u^{\prime} v^{\prime} / U_{\text {ref }}^{2}\right)_{\min @ x / c=0.8}+0.020\right] / 0.020
\end{gathered}
$$

RANS models have shown difficulties in predicting the size of the separation bubble as they tend to underpredict the turbulent shear stress in the separated shear layer, thus leading to a delayed reattachment. The separation bubble size in RANS is typically overpredicted by $35 \%$ to $38 \%$ depending on the turbulence model. More recently efforts have been made to improve the predictive capabilities of RANS solvers for smooth body separated flows by Rumsey et al. ${ }^{76,77}$ and Lardenau ${ }^{78}$ and overall good improvements have been achieved. However the reattachment location prediction remains unsatisfactory.

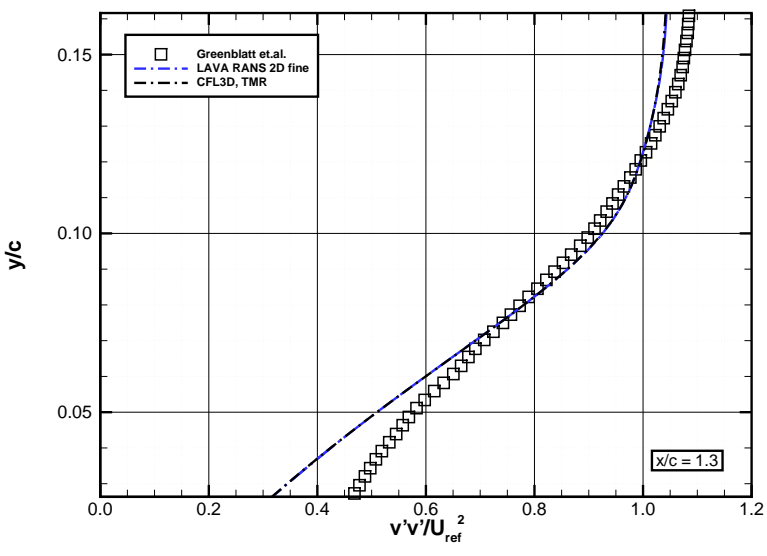

(a)

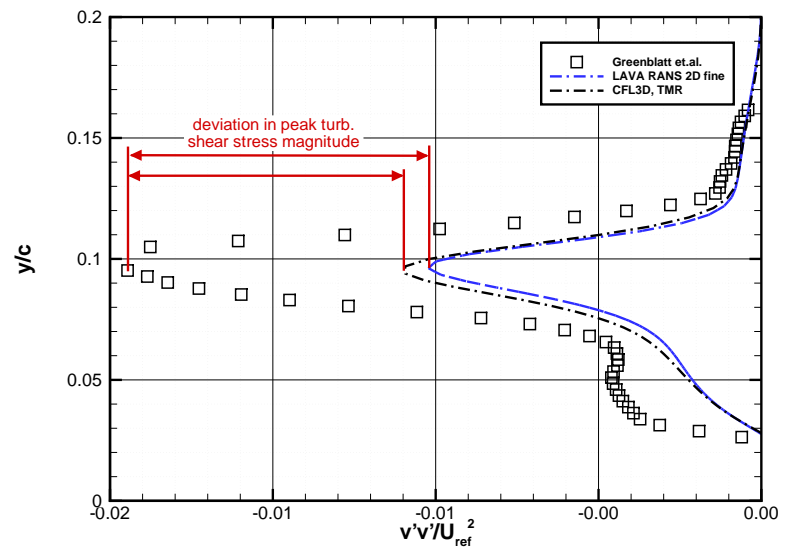

(b)

Figure 6: Comparison between RANS and Experiment for: (a) normalized velocity profile at x/c=1.3; (b) normalized shear stress at $\mathrm{x} / \mathrm{c}=0.8$

Figure 8a shows the axial velocity profiles at given $\mathrm{x} / \mathrm{c}$ locations and compares them with RANS results from CFL3D and experimental results from Greenblatt et al. ${ }^{1}$ An overall good agreement between the two different solvers and the three different mesh levels can be observed. The medium and fine mesh results are almost indistinguishable and consistent with CFL3D results. Table 1 shows the separation, reattachment locations as well as the bubble size for all discussed 2D simulations. The reattachment location is consistent between the two RANS solvers at around $1.26 \mathrm{c}$ and leads to a overprediction of the bubble size by approximately $38 \%$. This is in agreement with values reported in the literature for this configuration. Figure 6a shows a closeup of the normalized streamwise velocity at $\mathrm{x} / \mathrm{c}=1.3$ and demonstrates the overprediction of the bubble size with much smaller velocity values close to the wall. 
Figure 8b and Figure 6b show the normalized shear stress. A key issue that RANS solvers face for this flow configuration is the underprediction of the turbulent peak shear stress magnitude in the separated region resulting in insufficient turbulent mixing and thus a delayed reattachment. Utilizing the metrics defined in Eqn. 4 an underprediction of around $45 \%$ can be observed. The errors from all simulations are listed in Table 1.

Skin friction coefficient and pressure coefficient variations along the streamwise direction are shown in Figure 7 and compared with CFL3D and measurements. All grid levels compare reasonably well with experimental measurements in the upstream portion of the hump until separation occurs, a clear deficiency in predicting the reattachment location can be seen in the $C_{f}$ plot. As suggested in the TMR website, the pressure coefficient curve has been shifted by $\Delta C_{p}=-0.015$ in order to better match the experiments upstream. Overall the pressure coefficient is slightly underpredicted but matches the experiments reasonably well except inside of the recirculation region.

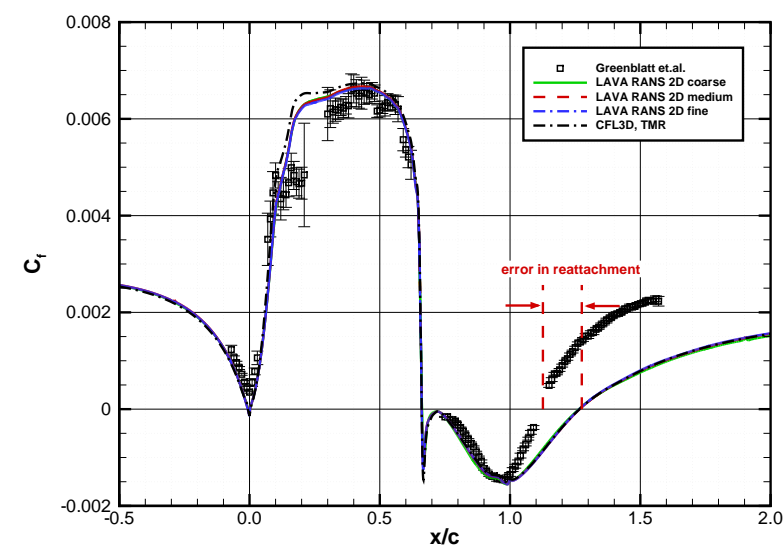

(a)

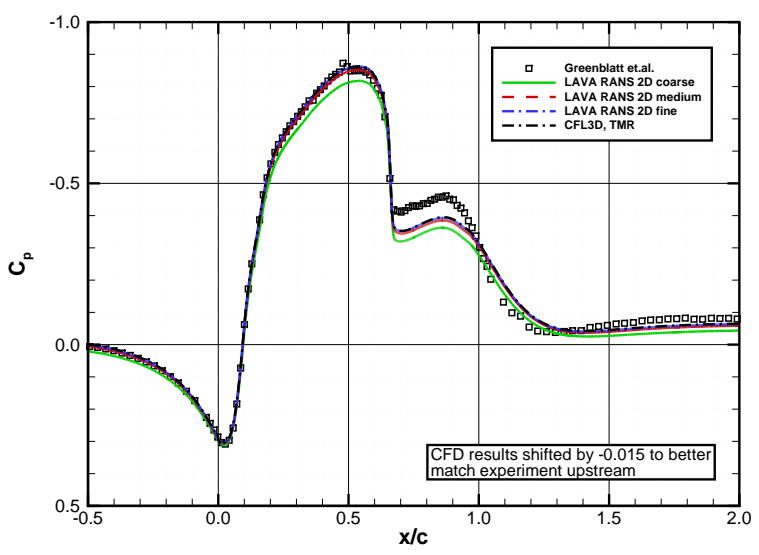

(b)

Figure 7: Comparison of skin friction coefficient $C_{f}$ (a) and pressure coefficient $C_{p}$ (b) with CFL3D and experiments.

In summary our RANS simulations agree very well with results reported in literature. The inability of RANS turbulence models to predict the separation bubble size accurately has been demonstrated. Clearly eddy resolving approaches are necessary to obtain improved results.

Table 1: Separation and reattachment locations and corresponding separation bubble size from 2D LAVA RANS simulations, CFL3D RANS simulations and 2D PIV centerline measurements of Greenblatt et al. ${ }^{1}$ Separation bubble error computed using Eqn. 3.

\begin{tabular}{lcccc}
\hline Case & $\begin{array}{c}\text { Separation location } \\
(\mathrm{x} / \mathrm{c})\end{array}$ & $\begin{array}{c}\text { Reattachment location } \\
(\mathrm{x} / \mathrm{c})\end{array}$ & $\begin{array}{c}\text { Bubble length } \\
(\Delta \mathrm{x} / \mathrm{c})\end{array}$ & $\begin{array}{c}\text { Error } \\
(\%)\end{array}$ \\
\hline Greenblatt et al. & $0.665 \pm 0.005$ & $1.10 \pm 0.005$ & 0.435 & - \\
CFL3D TMR & 0.661 & 1.263 & 0.602 & 38.3 \\
coarse & 0.662 & 1.260 & 0.598 & 37.4 \\
medium & 0.661 & 1.263 & 0.602 & 38.3 \\
fine & 0.661 & 1.263 & 0.602 & 38.3 \\
\hline
\end{tabular}

\section{IV.B. Hybrid RANS/LES - Structured Overset Grid System}

In the following section, results from our time-accurate structured curvilinear overset grid solver will be presented. Two different approaches have been chosen for this work and are described in Section III.A.1 for hybrid RANS/LES and in Section III.A.2 for WMLES (ZDES Mode 3).

The advantage of structured overset grids for wall bounded flows includes the ability to generate highly anisotropic grids to capture boundary layers and shear layers. Local refinement is used in the streamwise 


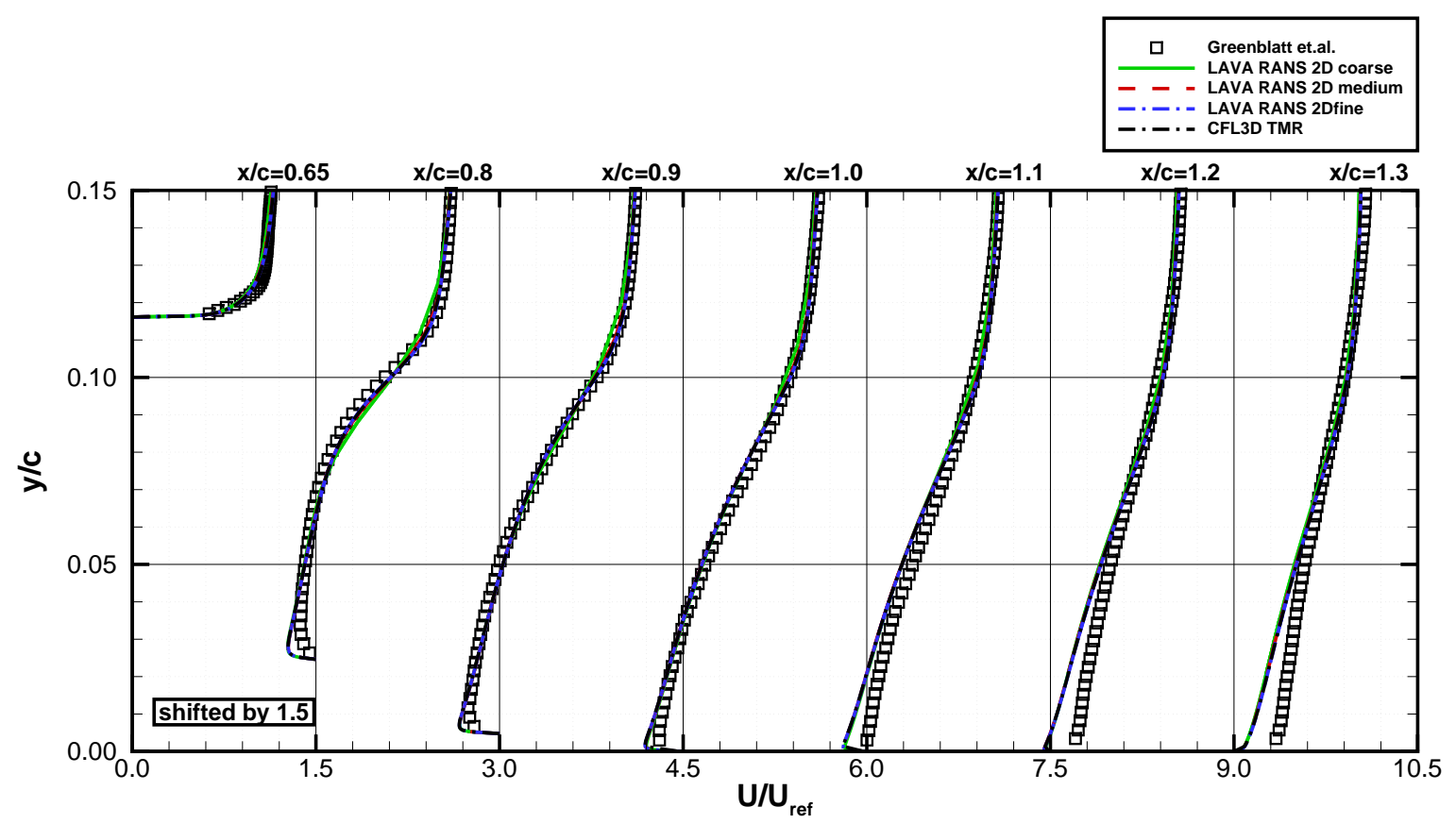

(a) profiles shifted by $\Delta U / U_{\text {ref }}=1.5$ along the horizontal axis

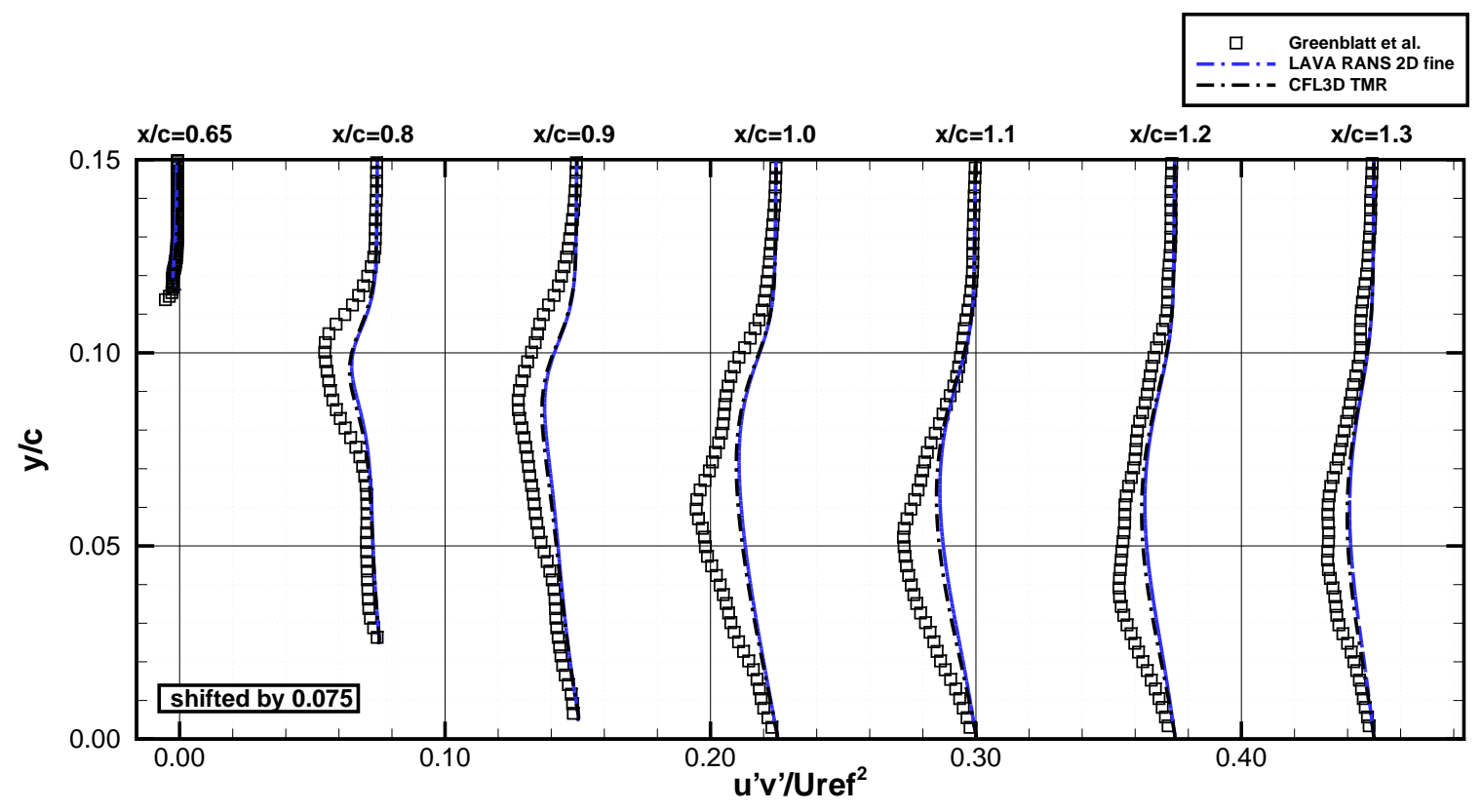

(b) profiles shifted by $\Delta u^{\prime} v^{\prime} / U_{r e f}^{2}=0.075$ along the horizontal axis

Figure 8: Comparison of (a) axial velocity profiles and (b) Reynolds shear stress with CFL3D and experimental results from Greenblat et al. taken from TMR. ${ }^{74}$ 
direction to capture the separation and reattachment locations more accurately. Tight-clustering is also used near the viscous walls to guarantee a wall normal resolution in viscous wall units of approximately one. The latter is a tremendous advantage when it comes to simulating wall-bounded flows compared to using Cartesian methods with isotropic cells. This will be discussed in more detail in section IV.C.

A time-step of $\Delta t=1.8 \times 10^{-5}$ seconds is used for the simulation along with 10 sub-iterations which equated to 3-4 orders of magnitude residual reduction at each physical time-step of the dual-time stepping algorithm. This time-step is equivalent to a $\Delta t^{+}=5.0$ and a $\frac{\Delta t U_{r e f}}{\delta_{0}}=0.02$. If an explicit time-marching scheme is used with the current mesh and a CFL restriction of unity is enforced, then the time-step would need to be reduced by three orders of magnitude compared to the current simulation because of the viscous wall spacing. To begin the simulations, steady RANS is performed until a converged solution is obtained. Next, the hybrid RANS/LES models are activated in selected regions and run for 10-15 convective time units in order to wash out transients. Finally, the simulation is restarted and turbulent statistics were collected over one or two full domain flow through times (FDFT), which corresponds to approximately 8 to 16 convective time units.

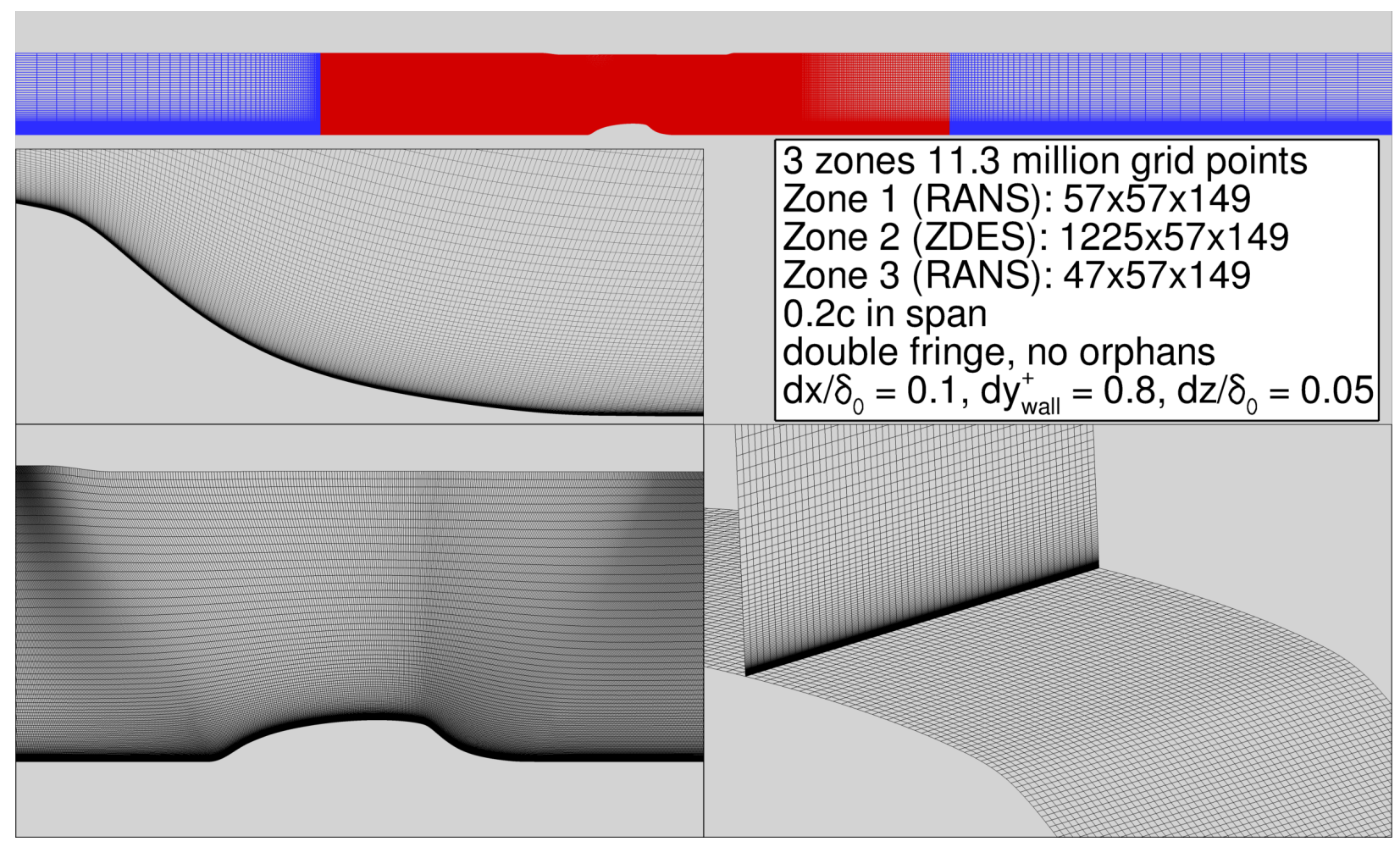

Figure 9: Mesh for unsteady curvilinear simulations. (top) blue zones are in pure RANS mode, red zones in hybrid RANS/LES or WMLES mode. Total number of grid points is 11.3 million. SEM seeding location at $\mathrm{x} / \mathrm{c}=-3.0$ (start of red zone). Measured $\delta_{0}$ at SEM seeding location.

The mesh was created following the suggestions of Deck ${ }^{35}$ for ZDES as well as our observations from the preliminary RANS study. Figure 9 shows a picture of the final mesh used in this work. The spanwise extent of the domain is 0.2 chord lengths. Previous studies by You et al. ${ }^{7}$ and Park ${ }^{79}$ have shown good agreement with a similar spanwise extent. The regions highlighted in blue mark zones in which pure RANS is used in order to provide upstream profiles, Reynolds stresses and to dampen reflections from the inlet and outlet in ZDES Mode 3. In order to insert meaningful turbulence at the interface between RANS and LES at $\mathrm{x} / \mathrm{c}=-3.0$ a modified version of Jarrin's ${ }^{69,70}$ Synthetic Eddy Method has been utilized (see Section III.C) for all ZDES runs and for DDES runs whenever mentioned.

Figure 10 shows the skin friction coefficient and pressure coefficient for RANS, DDES, DDES+SEM and ZDES Mode 3. In the DDES+SEM and ZDES Mode 3, synthetic turbulence was seeded at $x / c=-3.0$ using flow quantities from upstream RANS as an input. Upstream of the separation point the SA-DDES results are consistent with the SA-RANS results, indicating the attached boundary layer is staying in RANS mode as expected. The slight deviation between RANS and the hybrid models stems from small differences 

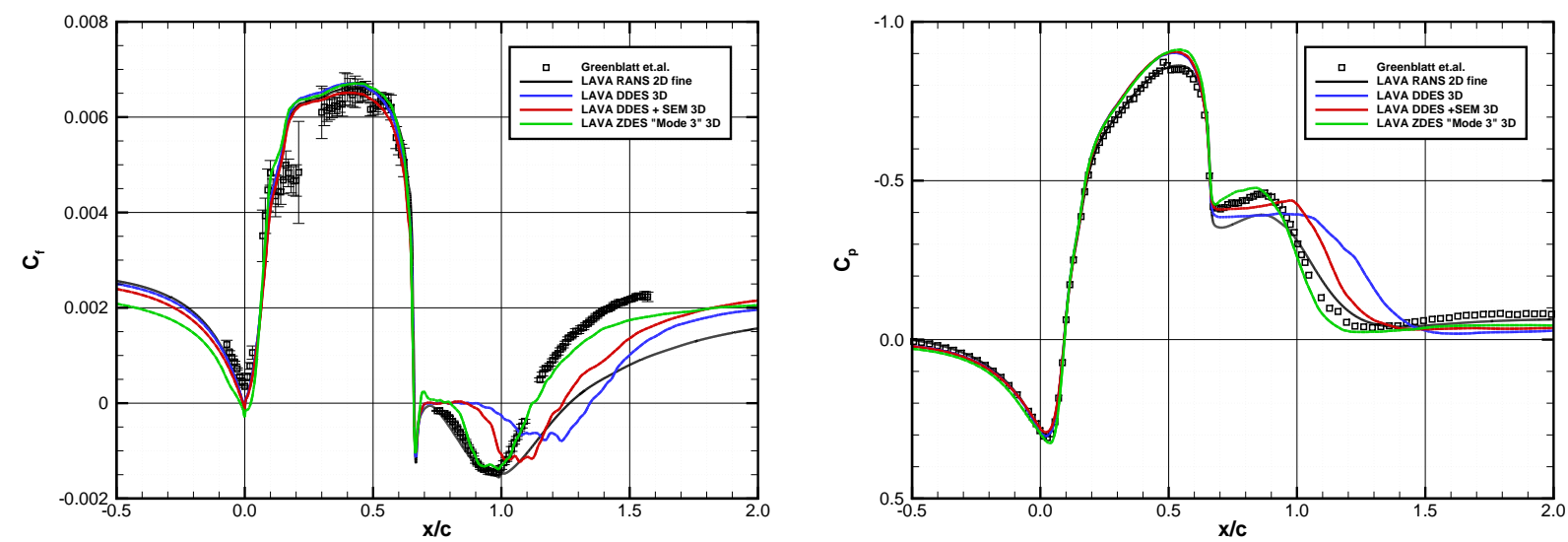

Figure 10: Comparison of skin friction coefficient $C_{f}$ (left) and pressure coefficient $C_{p}$ (right) between RANS, DDES, DDES+SEM and ZDES Mode 3. The SEM seeding location is at $x / c=-3.0$.

in the mesh. The DDES models recovered the RANS results up to the separation location. Downstream of the separation point the flow quickly reattaches near the wall creating a bifurcated separated flow pattern (see Figure 11) that is qualitatively different from what is observed in both the experiment and RANS simulations. The separated flow region is very well-predicted using SA-ZDES Mode 3 with almost no visible differences between the experiment and the computation.

The difficulties DDES models face for predicting shallow flow separation are associated with the gray area problem, which causes a delay in the development of three-dimensional structures in the shear layer. Similar problems have been observed by Garbaduk ${ }^{80,81}$ for SST-DDES and Probst et al. ${ }^{81}$ SA-DDES, where the latter seems to suffer even more for this configuration. As seen in Figure 14 and Figure 15 depicting snapshots of vorticity magnitude and turbulent eddy viscosity, SA-DDES suffers from a strong delay in the development of 3D structures in the separated shear layer, even though it appears to transition to LES mode almost immediately downstream of the separation location (as indicated by the $f_{d}$ function in Figure 15 (left)). This behavior persists for SA-DDES even when using the improved length scale. ${ }^{16,36}$ A similar behavior for DDES was recently reported for axial round jets by the authors. ${ }^{82}$ Seeding turbulent structures using SEM improves the transition to a certain degree, as turbulent structures upstream of the separation point are resolved and not modeled in the outer edge of the boundary layer.

This outer shear layer separation overprediction, as well as the inner layer reattachment, is also reflected in the velocity profiles in Figure 12. The inner-layer reattachment occurs between $x / c=0.68$ and $x / c=0.89$ for the DDES models. Errors due to the 2D structures in the shear layer are prominent for all profiles shown and improvements from the turbulent structures created by the SEM can also be seen. Another interesting observation is that adding SEM structures changes the skin friction upstream of the separation. Similar behavior is observed when using SEM in a Zero pressure gradient flat plate simulation, where the skin friction drops and a certain development length is necessary in order to recover from this (see Jarrin et al. ${ }^{69,70}$ ).

The turbulent eddy viscosity from the ZDES Mode 3 at $h_{w}=0.1 \delta_{0}$ decays much quicker compared to the DDES runs and only maintains an eddy viscosity ratio of around 5 to 8 in the shear layer. This difference becomes even more prominent when looking at an iso-contour of the Q-criterion ${ }^{\mathrm{c}}$ colored by the streamwisevelocity illustrated in Figure 13. Highly correlated structures in spanwise direction can be observed for the DDES model whereas the ZDES Mode 3 model has realistic 3D turbulent structures upstream of the separation point, as expected.

\section{IV.B.1. ZDES Mode 3 - influence of interface location}

In this section we will study the effects of the interface location $h_{w}$ between RANS (which serves as a wallmodel in ZDES Mode 3) and the outer LES simulation. According to Deck ${ }^{42}$ and Renard and Deck ${ }^{43}$ it is

\footnotetext{
${ }^{\mathrm{c}} \mathrm{Q}$-criterion: second invariant of the velocity-gradient tensor. $Q=\frac{1}{2}\left(\Omega_{i j} \Omega_{i j}-S_{i j} S_{i j}\right)$ where $\Omega_{i j}$ is the vorticity tensor and $S_{i j}$ is the strain-rate tensor.
} 

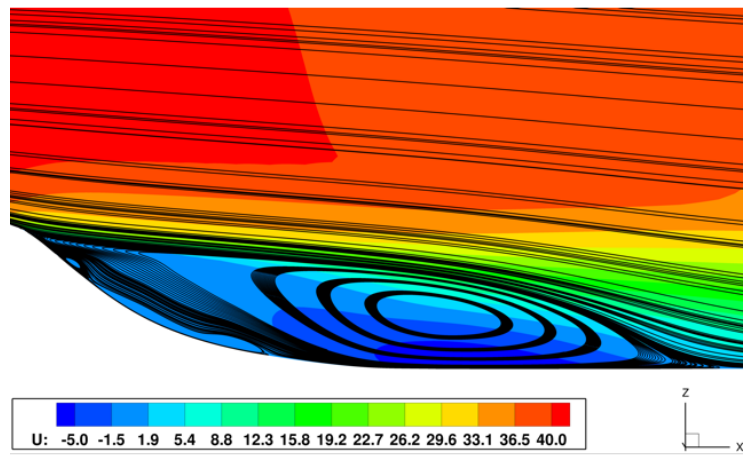

Figure 11: Contour plot of averaged streamwise velocity component from DDES simulation. Streamtraces show bifurcated flow pattern within the recirculation bubble shortly after the separation.

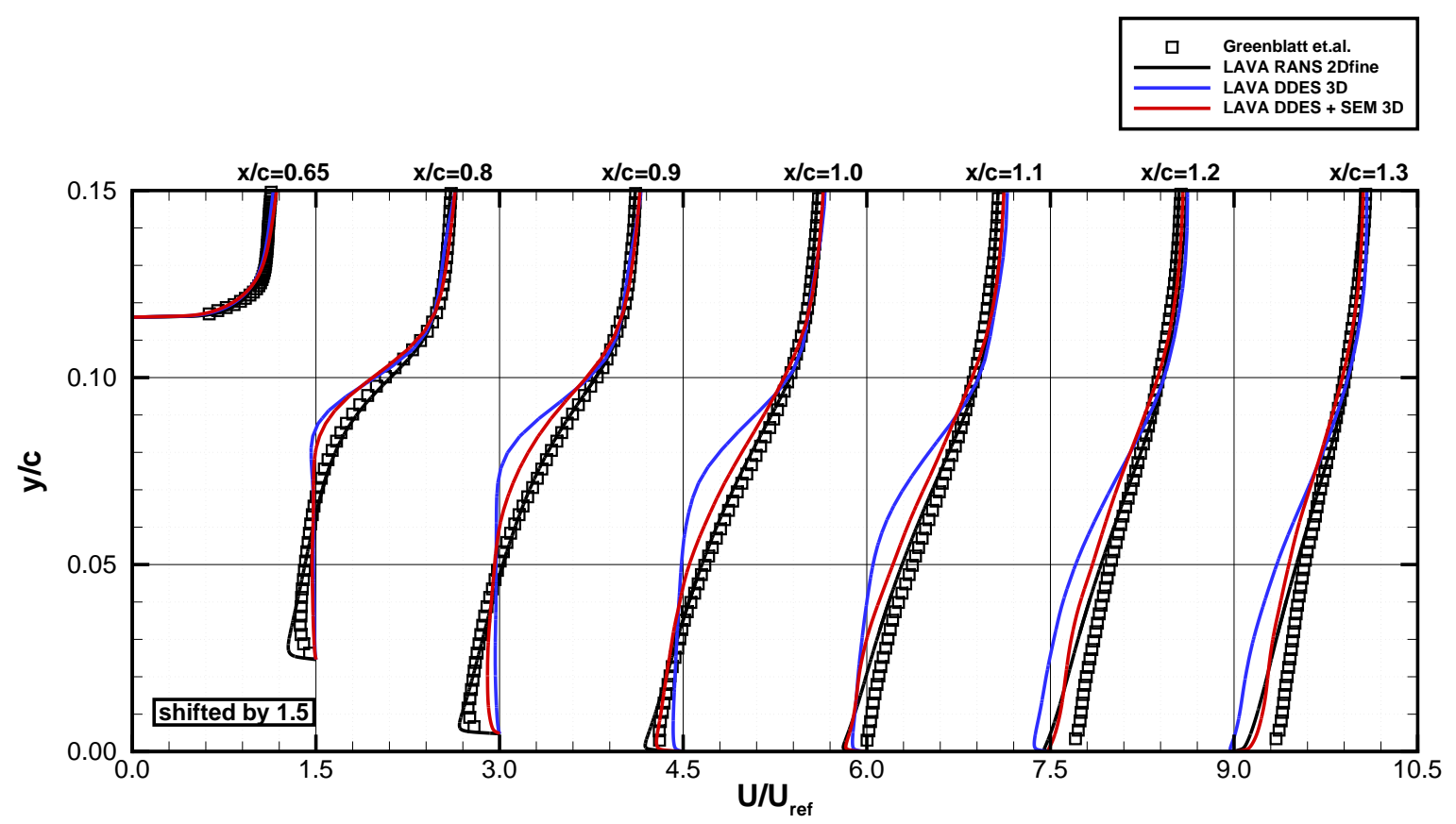

Figure 12: Normalized axial velocity profiles at given $\mathrm{x} / \mathrm{c}$ locations for DDES and DDES+SEM. Inner layer reattachment between $\mathrm{x} / \mathrm{c}=0.68$ and $\mathrm{x} / \mathrm{c}=0.89$. Outer-layer overprediction due to large $2 \mathrm{D}$ structures in shear layer. Profiles succesively shifted by $\Delta U / U_{\text {ref }}=1.5$
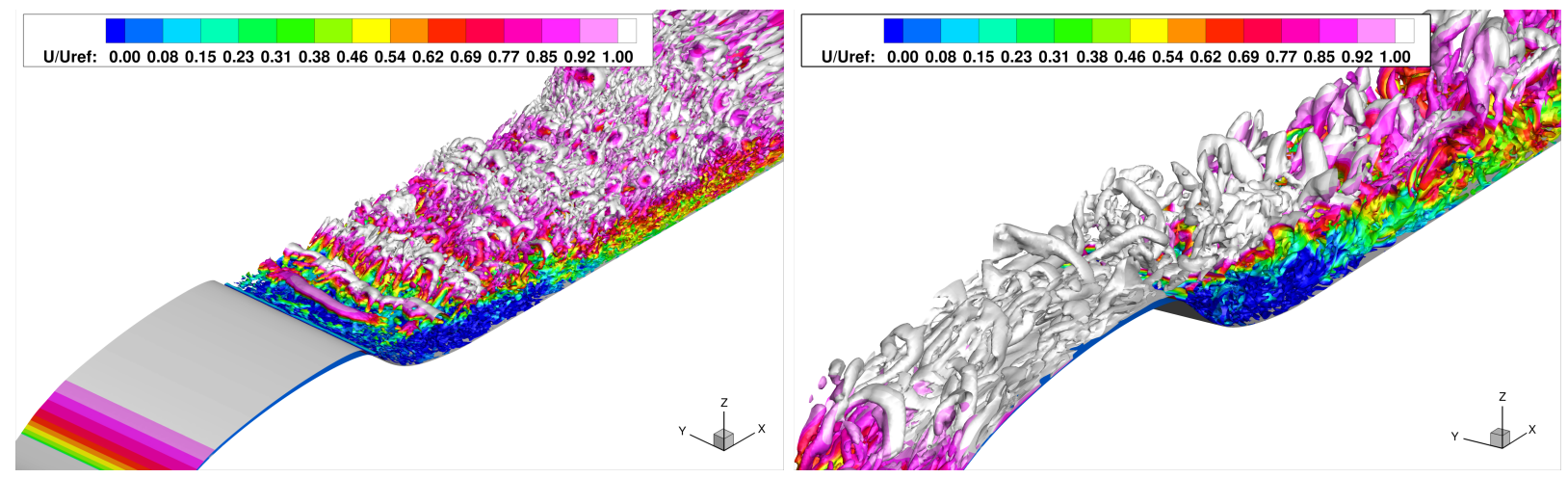

Figure 13: Iso-contour of Q-criterion colored by normalized streamwise velocity: (left) DDES, (right) ZDES Mode 3. Strong correlation in streamwise direction for DDES past the separation location. 
(a) DDES

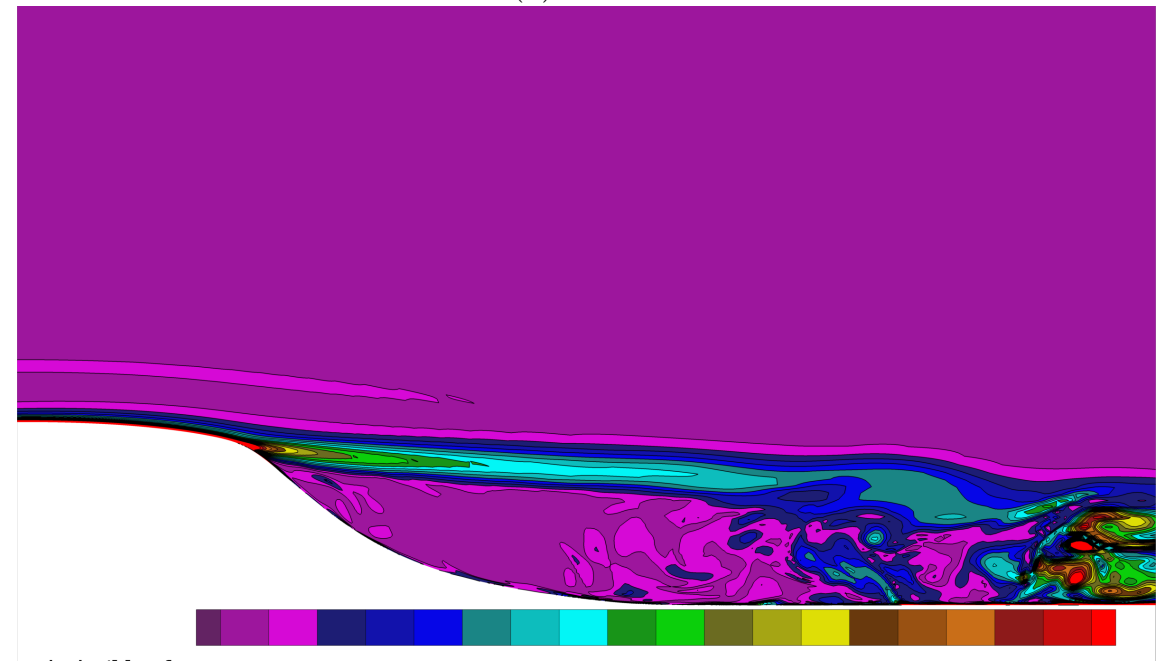

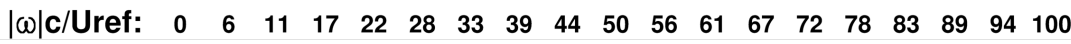

(b) DDES + SEM

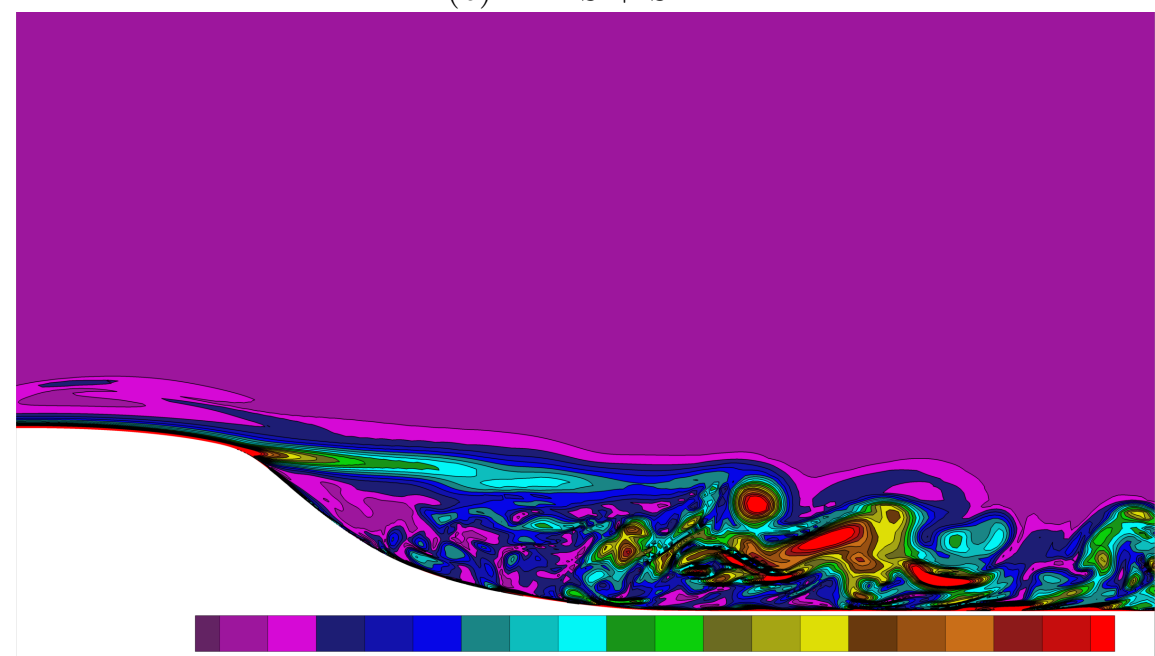

$\begin{array}{llllllllllllllllllll}|\omega| \text { c/Uref: } & 0 & 6 & 11 & 17 & 22 & 28 & 33 & 39 & 44 & 50 & 56 & 61 & 67 & 72 & 78 & 83 & 89 & 94 & 100\end{array}$

(c) ZDES "Mode 3" with interface at $0.1 \delta_{0}$

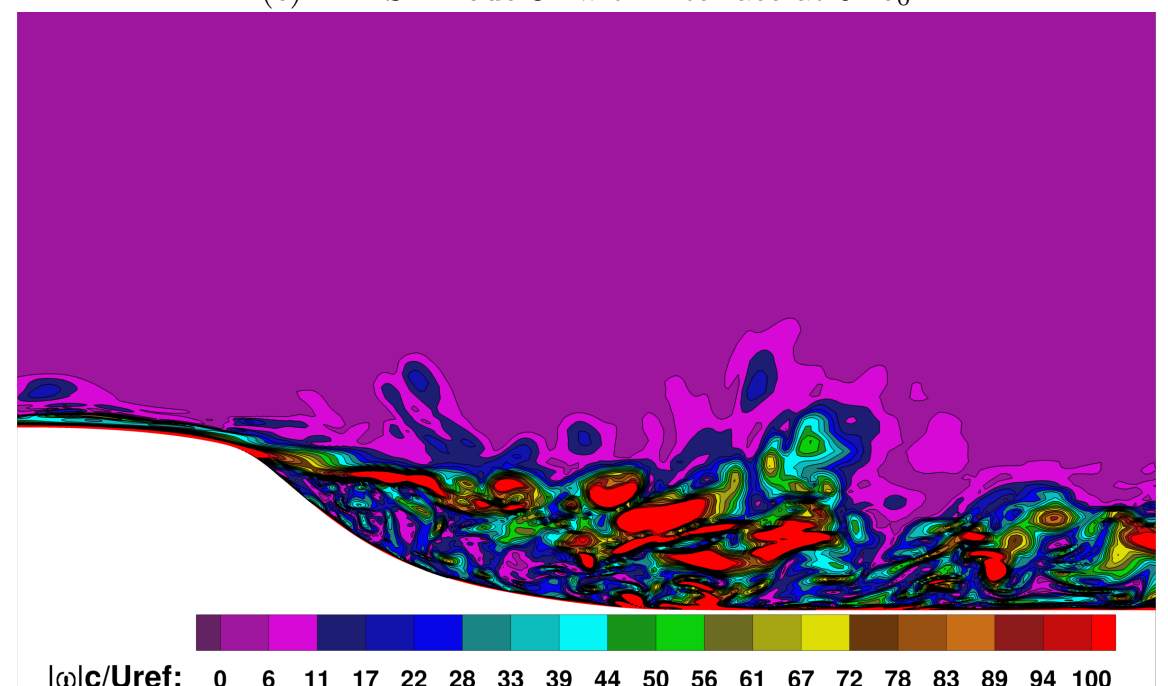

Figure 14: Snapshot of the normalized vorticity magnitude in the xy-plane. 
(a) DDES
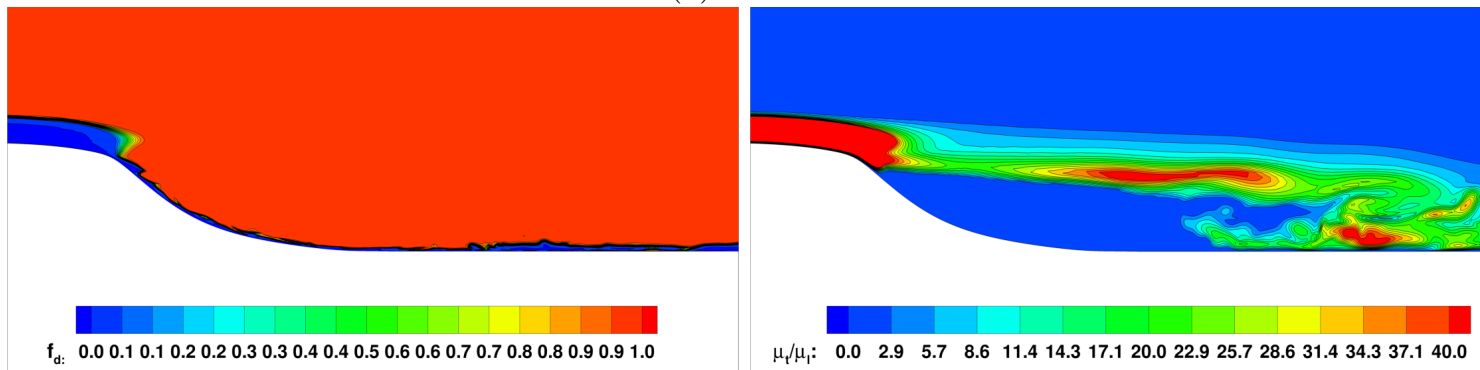

(b) DDES + SEM

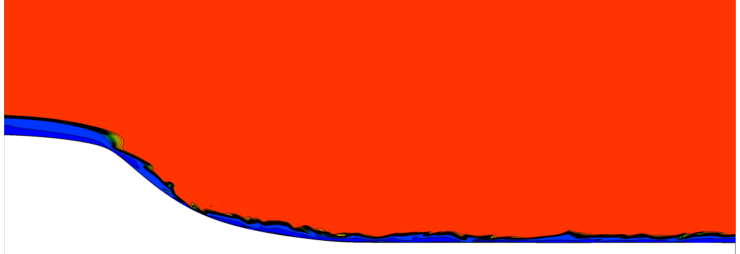

$f_{d:} \quad 0.00 .10 .10 .20 .20 .30 .30 .40 .40 .50 .60 .60 .70 .70 .80 .80 .90 .91 .0$

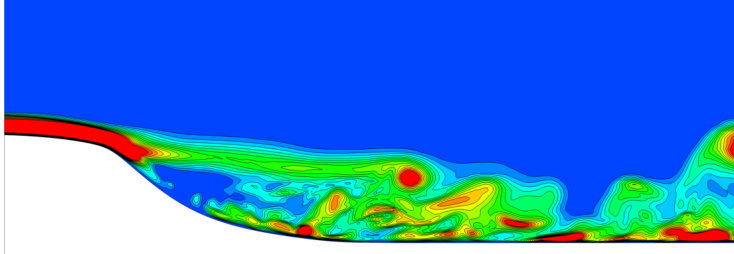

$\mu_{\mathrm{t}} / \mu_{1}: \quad 0.0 \quad 2.95 .7 \quad 8.6 \quad 11.414 .317 .120 .022 .925 .728 .631 .434 .337 .140 .0$

(c) ZDES "Mode 3 " interface $h_{w}=0.1 \delta_{0}$

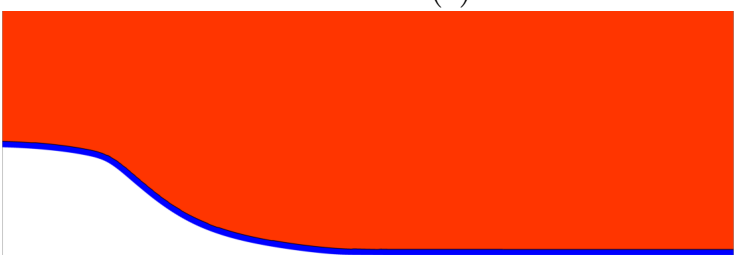

0.00 .10 .10 .20 .20 .30 .30 .40 .40 .50 .60 .60 .70 .70 .80 .80 .90 .91 .0

(d) ZDES "Mode 3" interfce $h_{w}=0.3 \delta_{0}$
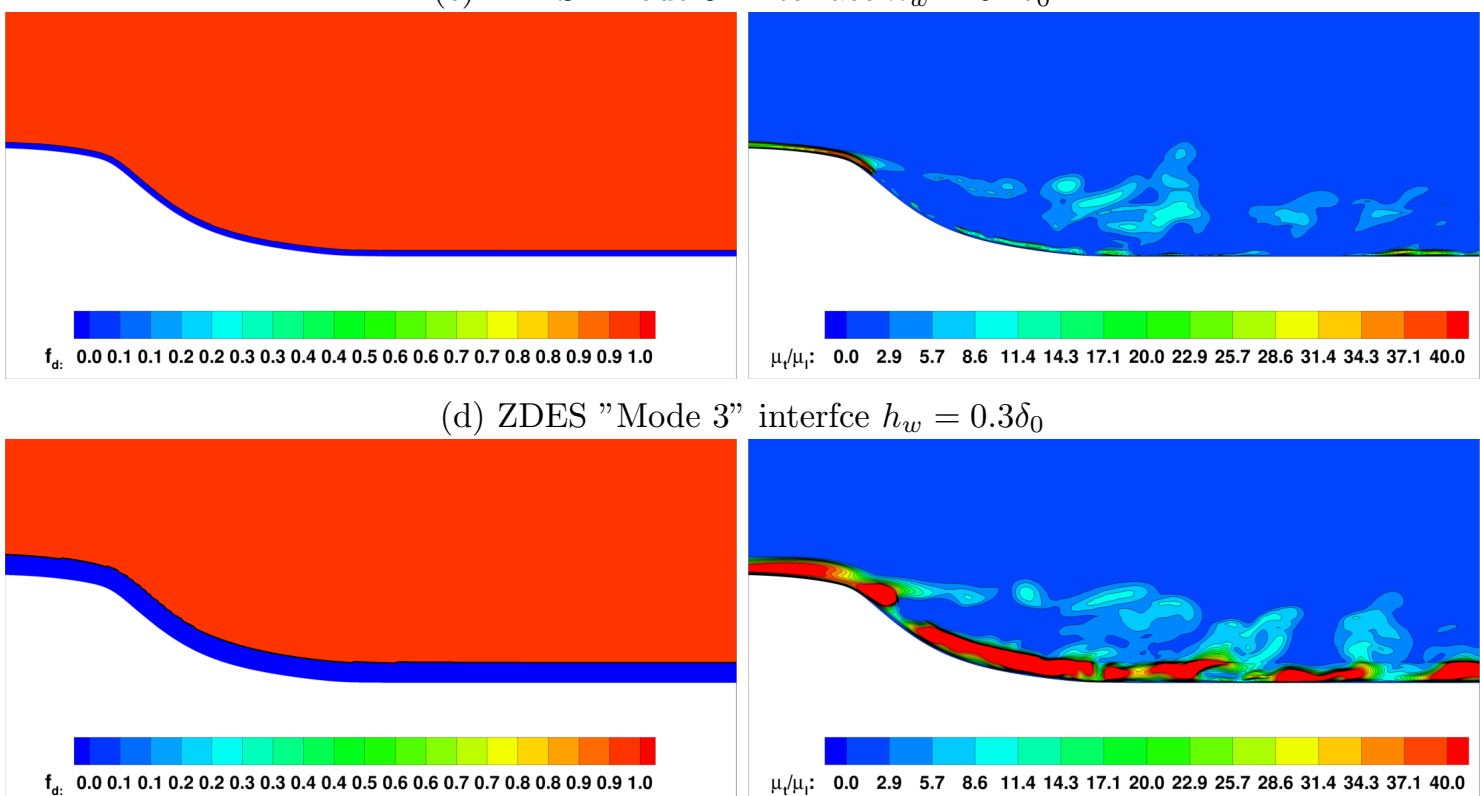

Figure 15: Comparison of four different hybrid RANS/LES simulations: (left) shielding function $f_{d}$; (right) turbulent eddy viscosity ratio $\mu_{t} / \mu_{l}$ 
recommended to constrain the interface location between inner layer RANS and outer layer LES in boundary layer thickness units. They observed that this choice led to a better skin friction prediction, for a given grid resolution.

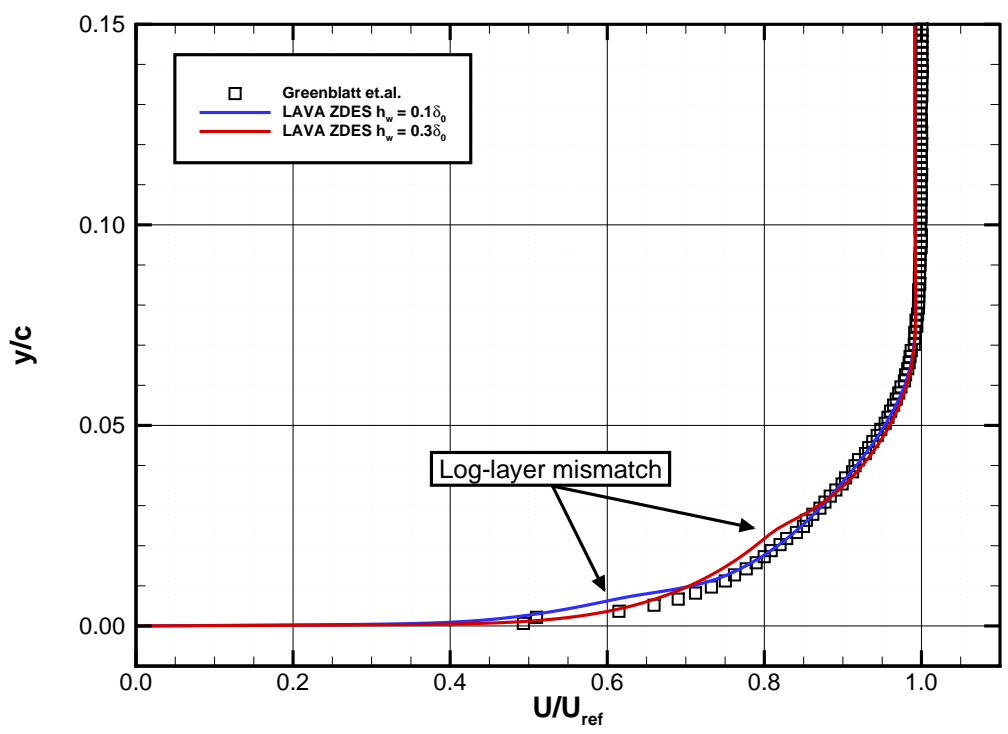

Figure 16: Inflow profile with ZDES for different interface locations: (blue line) $h_{w}=0.1 \delta_{0}$ and (red line) $h_{w}=0.3 \delta_{0}$.

Before investigating the influence of the interface location on the integrated quantities $C_{f}$ and $C_{p}$ we assess if both interface locations reproduce the experimental inflow profile at $x / c=-2.14$. It should be noted that the interface location used in our simulation is at a fixed height throughout the whole domain. Two different interface locations are considered, one at $h_{w}=0.1 \delta_{0}$ and the second at $h_{w}=0.3 \delta_{0}$, where $\delta_{0}$ is the boundary layer thickness at the inflow plane. Figure 16 shows the time-averaged normalized velocity profile at this location. Both simulations reproduce the velocity profile with good agreement. A clear log-layer mismatch can be seen for both interface locations.

Skin friction and pressure coefficient distributions for the two different interface locations are depicted in Figure 17. As we used a constant wall distance for the interface location in our simulations, the actual value of $h_{w} / \delta$ can locally be larger or smaller than 0.1 or 0.3 . This is indeed the case in the region between $-0.5<x / c<0.1$ where the boundary layer is thicker. In this region the larger interface location leads to a better agreement. Opposite behavior can be observed right before the separation location, where the boundary layer is thinner. In order to have a "smarter" way of determining the interface location, an effort is being made to implement a sensor based on the local BL thickness.

In Figure 18 the wall normal Reynolds stress component for both interface locations are compared to Greenblatt's data. Within the recirculation bubble $(x / c=0.8,0.9)$ better agreement is obtained for $h_{w}=0.3 \delta_{0}$. As soon as the boundary layer starts reattaching, better agreement with measurements is achieved for the interface location closer to the wall.

\section{IV.B.2. ZDES "Mode 3" - timestep sensitivity}

The influence of the timestep size as well as the total time the solution is averaged over is examined in this section. Three different timestep sizes are considered. Figure 19a shows the dependence of the number of timesteps the solution is averaged over. 1 Full domain flow through time consists of approximately 8 convective time units. A slight difference can be seen in the skin friction, especially in the adverse pressure gradient region and the recovery region leeward of the hump. This indicates that averaging over only 8 to 10 CFTs is not sufficient. We now examine the sensitivity of the results to the timestep size. For this purpose three different simulations with timesteps of $\Delta t^{+}=5.0,1.0$ and 0.5 have been conducted. Figure $19 \mathrm{~b}$ and Figure 20 depict the skin friction coefficient and the Reynolds stress component $u^{\prime} v^{\prime}$. The latter has been 

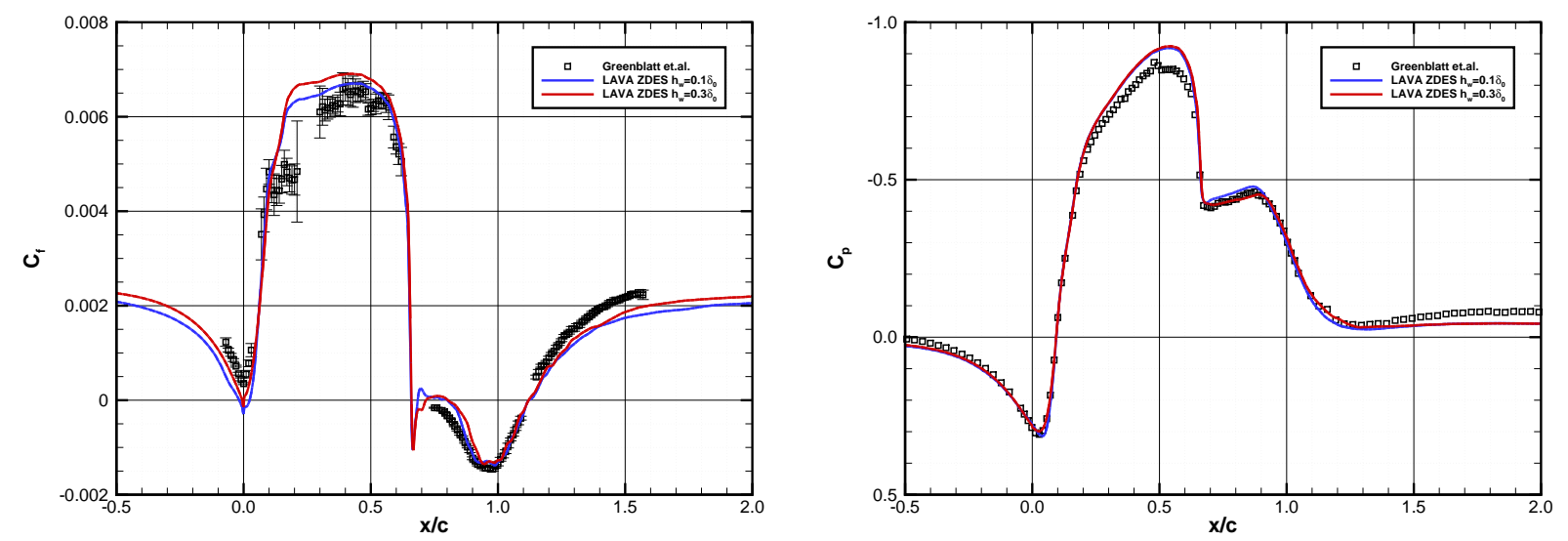

Figure 17: Effect of the RANS/LES interface $h_{w}$ location on the skin friction coefficient and pressure coefficient. Two different locations at $h_{w}=0.1 \delta_{0}$ and $h_{w}=0.3 \delta_{0}$ are considered.

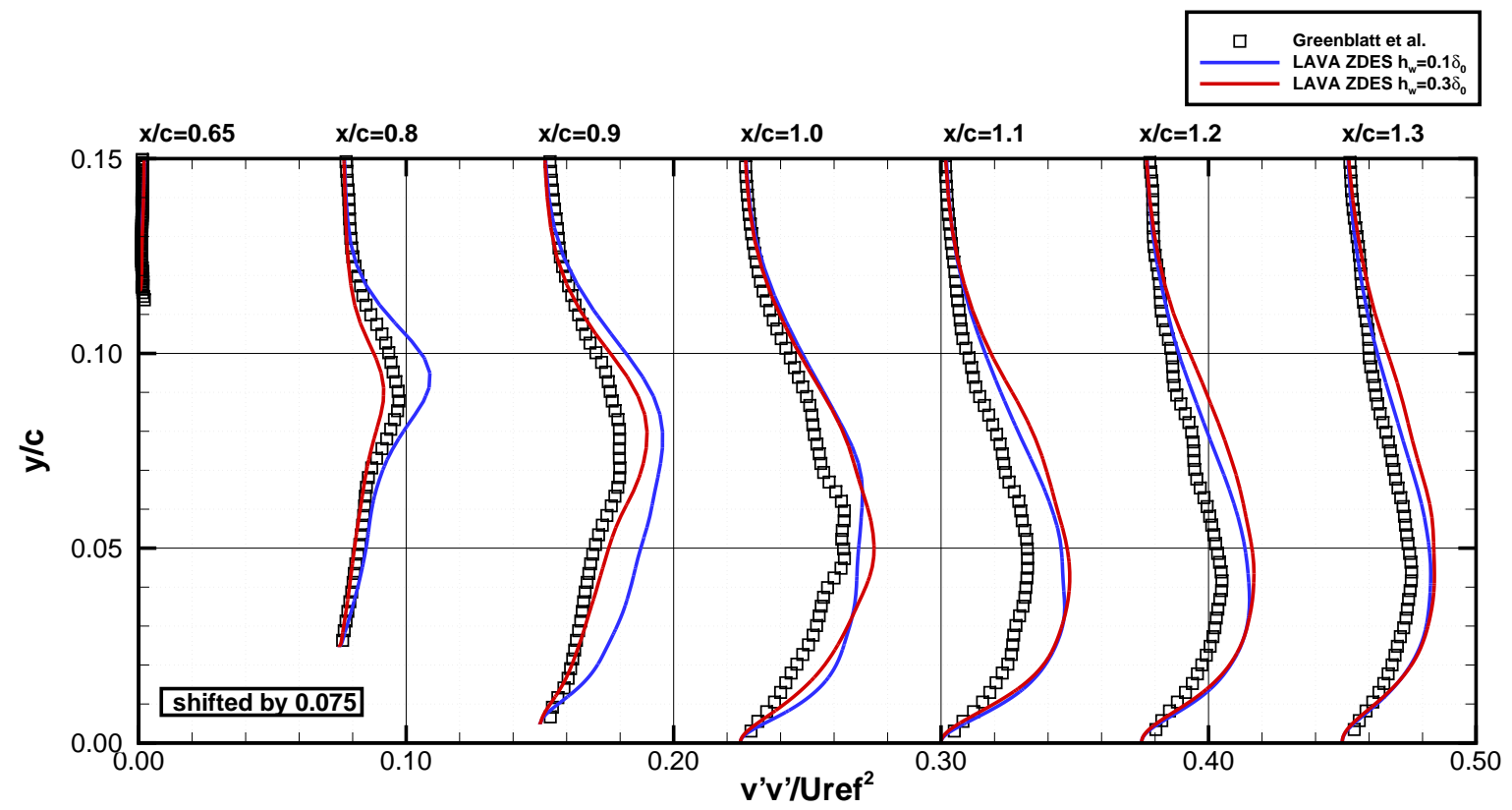

Figure 18: Variation of Reynolds stress $v^{\prime} v^{\prime}$ along seven different $x / c$ locations. Profiles successively shifted by $\Delta v^{\prime} v^{\prime}=0.075$ along the horizontal axis. 
chosen as it showed the biggest impact of timestep size on the overall results. For all timesteps full domain flow throughs have been simulated. The smallest timestep does the best job in predicting the skin friction, however it appears that the larger timestep does a better job in matching the $u^{\prime} v^{\prime}$ component inside of the separation bubble. This is possibly due to a fortuitous cancellation of multiple errors, but further investigation is required. Overall good agreement was observed with all selected timestep sizes.

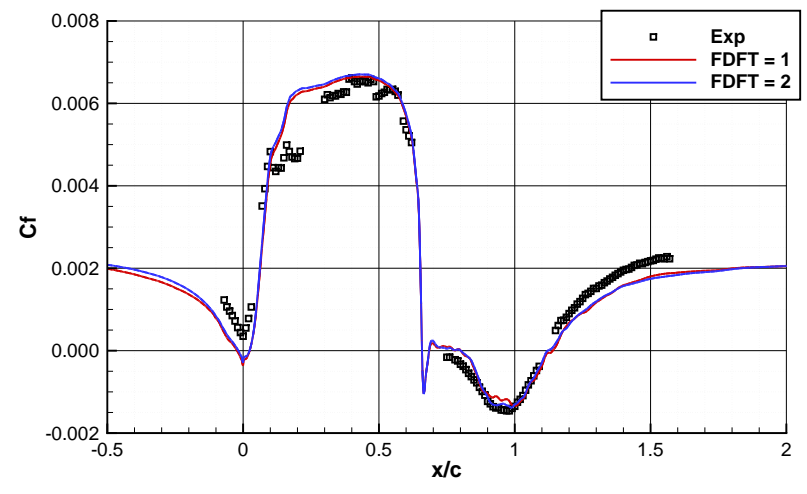

(a)

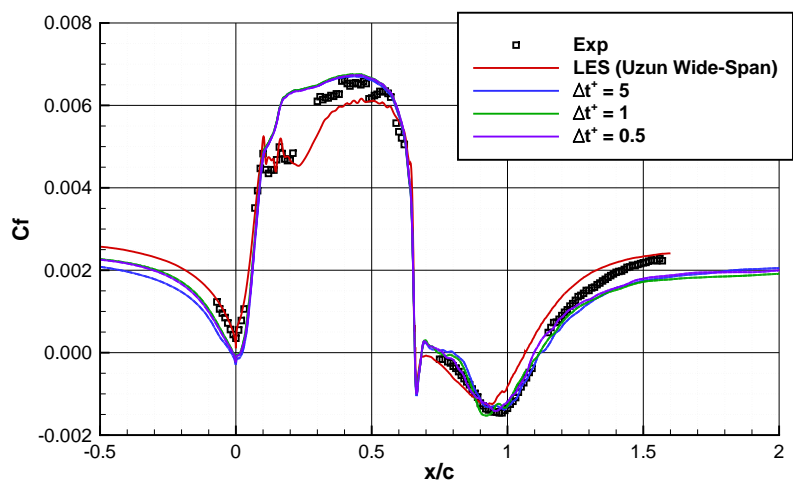

(b)

Figure 19: Comparison of skin friction coefficient: (a) 1 vs 2 Full Domain Flow throughs (FDFT). 1FDFT corresponds to around 8 convective flow throughs. (a) timestep sensitivity study with 3 different time step sizes of $\Delta t^{+}=0.5,1.0$ and 5.0. Data from wall resolved LES from Uzun et al. is plotted for comparison.

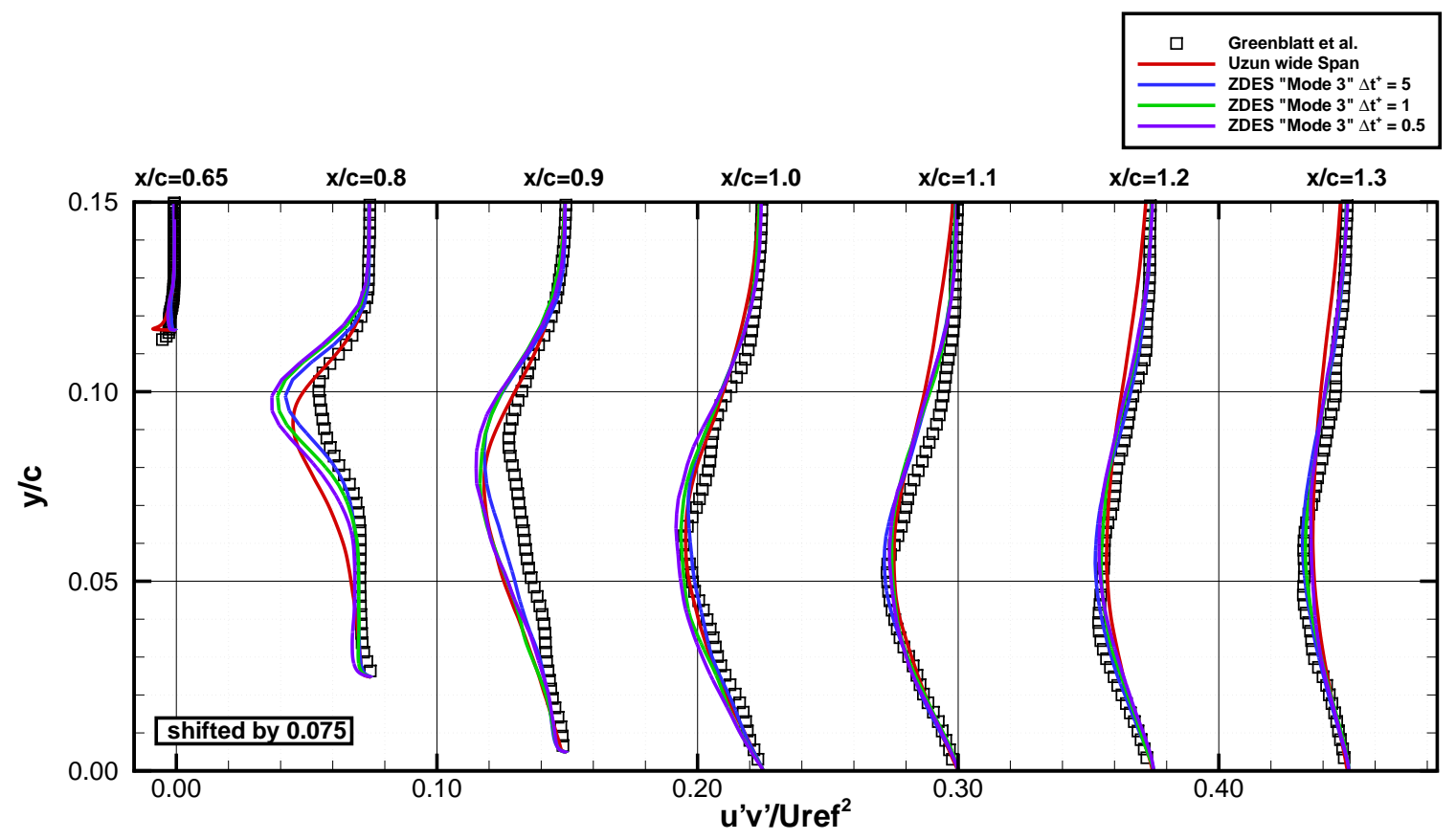

Figure 20: Reynolds stress profile of the $u^{\prime} v^{\prime}$ component at seven different axial locations. Three different timestep sizes considered $\Delta t^{+}=5.0,1.0,0.5$. Profiles successively shifted by $\Delta u^{\prime} v^{\prime}=0.075$.

\section{IV.C. Comparison of LBM-LES with ZDES Mode 3 and results from literature}

This section will discuss results obtained with our Lattice Boltzmann solver in LES mode. A comparison will be made with ZDES Mode 3 and selected results from literature. For our comparison we selected wall resolved LES simulations on a wide-span configuration $\left(L_{z}=0.4 c\right)$ from Uzun and Malik ${ }^{3}$ who used a mesh 
consisting of 420M cells with $y^{+}<1$. We also compare our results with LBM Very Large Eddy Simulation (LBM-VLES) of Duda and Fares. ${ }^{4}$ This approach is characterized by adding modeled turbulence in under resolved areas, and is comparable to a hybrid RANS/LES approach. Two different configurations were used in their study, one with periodic boundary conditions and a spanwise extent of $0.75 c$. A second simulation was conducted where side walls and tunnel geometry were included to account for blockage effects. If not explicitly stated, their results with side walls are used for comparison. A mesh size of $149.8 \mathrm{M}$ cells with a maximum resolution in viscous wall units of 75 was reported.
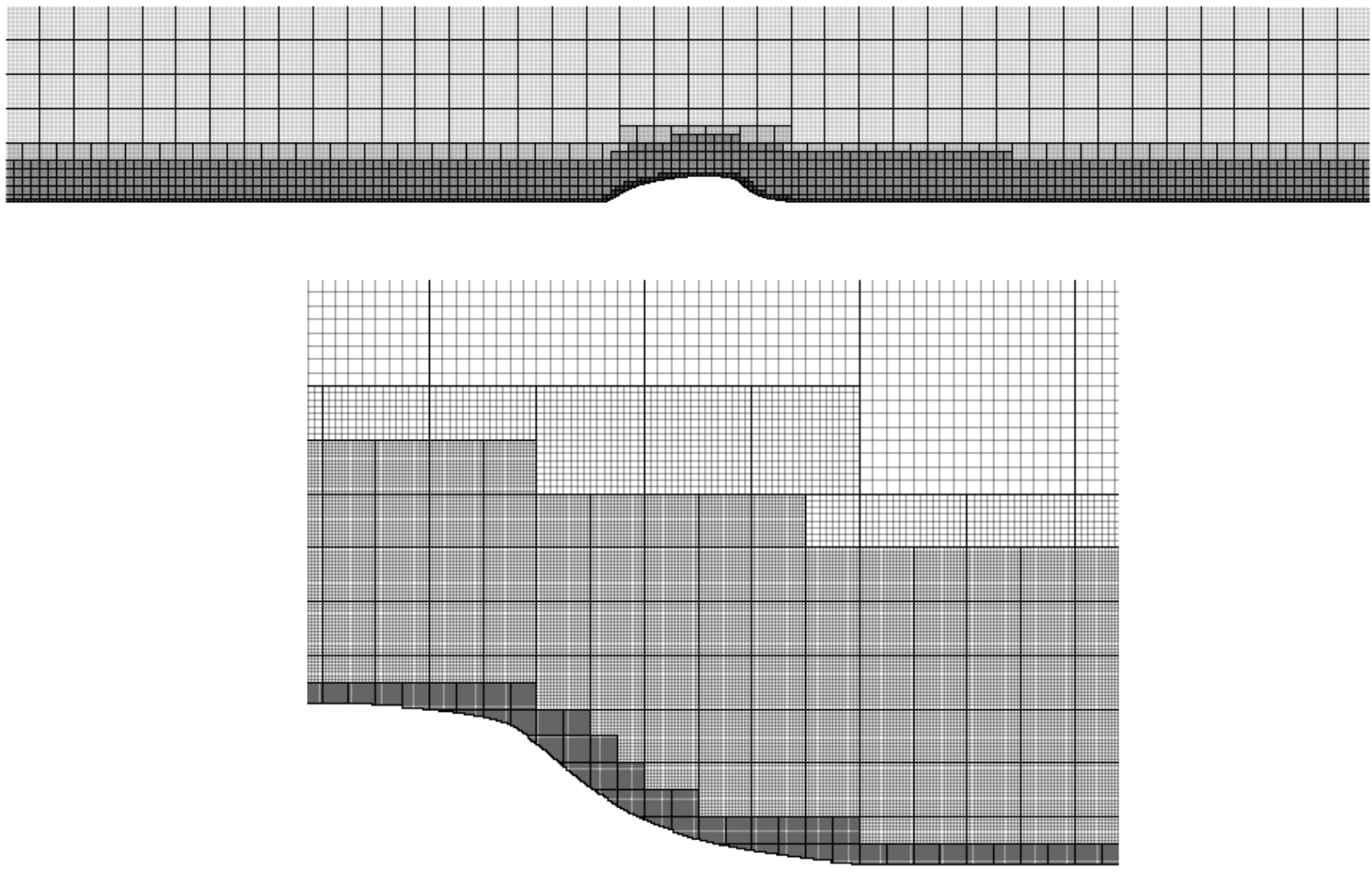

Figure 21: Cartesian mesh with total of 5 levels of mesh refinement. Spanwise extend $0.2 \mathrm{c}$ with $y^{+}$-value smaller than 50 around the hump. Level 3 in regions with high vorticity. Finest level very close to the wall turned off for visualization purpose.

The computational mesh used for our LBM simulations is depicted in Figure 21. A total of five levels of mesh refinement with a ratio of 2:1 was used, as spatial resolution requirements significantly differ within the domain. The finest level is used in the near wall region around the hump ranging from $x / c=-0.2$ to $x / c=1.3$, all other viscous walls are modeled with the second finest level. In regions of high vorticity the third finest level is used and extended all the way to where the SEM is seeded. All the LBM-LES simulations presented in this paper were performed using the entropic multi-relaxation time model on the D3Q27 lattice. Periodic boundary conditions were applied in the spanwise direction. Velocity and turbulent quantities at the inflow boundary located at $x / c=-3.0$ were specified based on our precursor RANS-SA simulation which was carried out to match the experimental value of the momentum thickness based Reynolds number $R e_{\theta}=7200$. The correlation length $\sigma_{S E M}$ needed for the SEM is estimated based on Bradshaw's hypothesis using Eqn. 2. Additional simulations have been carried out with a single value for the correlation length in the SEM of $\sigma_{S E M}=0.15 \delta_{0}$. Only minor differences have been observed once realistic turbulent structures were developed. Preliminary studies of this turbulent inflow condition using hybrid RANS/LES have shown 
that a development length of at least $20 \delta_{0}$ is required to develop fully turbulent structures. Additional turbulent inflow conditions that recover skin friction distribution faster are currently under investigation within the LAVA framework (e.g. Shur et al. ${ }^{71}$ ).

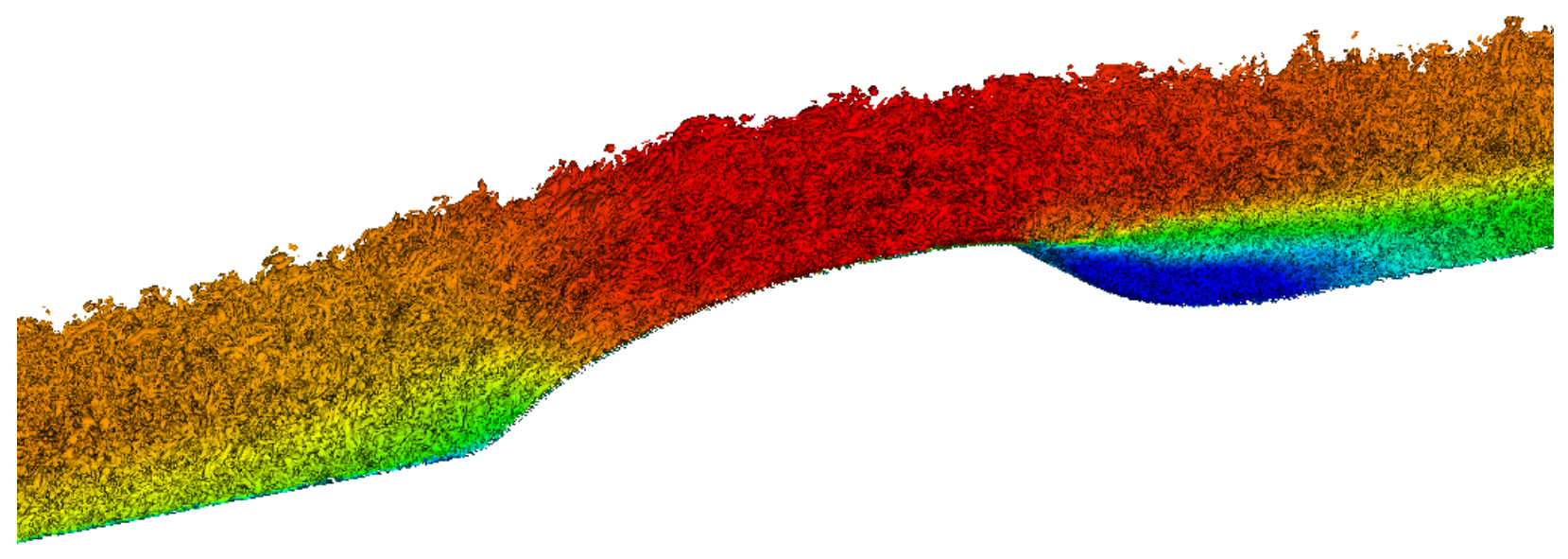

Figure 22: Iso-contour of Q-criterion coloured by normalized streamwise velocity from LBM-LES.

Due to the explicit nature of the numerical method, and the recursive algorithm outlined in Figure 3 the overall timestep is linked to the size of the cell on the finest level, with an acoustic CFL constraint. Therefore a timestep of $\Delta t=4.6 \times 10^{-7}$ seconds is used and the simulation is run for a total physical time period of 0.45 seconds which corresponds to around 33 convective time units. The first 5 convective time units were discarded in order to wash out transients. Figure 22 shows an instantaneous snapshot of an isosurface of Q-criterion colored by the normalized streamwise velocity to depict the vortical features and fine scales captured by the simulation. Much finer 3D turbulent structures can be observed compared to our previous hybrid RANS/LES simulations. This stems from the larger number of cells used, which were required because of the isotropic nature of the cells.

Axial velocity profiles for ZDES, LBM-LES, WRLES ${ }^{3}$ and LBM-VLES $^{4}$ (full span and periodic) are plotted in Figure 23 at seven different streamwise locations. The first measurement station is just upstream of the separation location at $x / c=0.65$. The measurements at $x / c=1.1,1.2$ and 1.3 show reattachment and recovery of the flow. Additionally, three stations within the separation bubble at $x / c=0.8,0.9,1.0$ are depicted. Overall, excellent agreement can be observed with measurements and other simulation results from literature. The velocity profiles near the apex of the hump are slightly overpredicted for the ZDES and WRLES simulations and underpredicted for the LBM simulations. However it is noted that the experimental PIV measurements at this location, where the boundary layer is very thin, are not sufficiently accurate in the near wall region. ${ }^{8}$ Interestingly, as shown in Figure 8, RANS simulations predict the Reynolds-stresses at this location very well, which might be a reason why LBM-VLES and ZDES perform significantly better, as both models utilize the modeled stress components from a RANS turbulence model. Once the flow has separated, RANS severely underpredicts Reynolds stresses in the shear layer that originates from the separation point. This is a well-known deficiency of state-of-the-art RANS turbulence models in regions where the underlying flow is far from equilibrium and remains an extremely active area of research. ${ }^{77,78}$

Figures 24-26 show the mean Reynolds stress comparison at the same measurements locations. The RMS profiles in the simulations appear to be more energetic compared to experimental measurements. This is especially prominent very close to the separation location for both of the simulations in LES mode where no modeled stress is present. The over prediction of the Reynolds stresses further away from the wall in our LAVA LBM-LES simulations is likely due to the consideration of blockage effects. Unfortunately no RMS data from the periodic setup was reported by Duda and Fares. ${ }^{4}$ However, numerical experiments conducted by Uzun and Malik ${ }^{8}$ modifying the contoured top wall seem to support our conjecture. The influence of the spanwise extent of the simulation domain needs to be evaluated more carefully. 
Table 2 lists the separation as well as reattachment location and the corresponding bubble size for all simulations and gives an error estimate based on Eqn. 3. Our LAVA LBM-LES and ZDES "Mode 3" show an improvement of approximately $90 \%$ in predicting the separation bubble size, thereby meeting NASA's RCA technical challenge of reducing the the predictive error by at least $40 \%$. The DDES approach with improved length scales used in this work ${ }^{16}$ significantly overpredicted the separation bubble size due to the well known problem of modeled-stress depletion which delays the development of 3D turbulent structures in the separated shear layer. Efforts to mitigate this issue by replacing the length scale definition in the model with a shear layer adapted version of the subgrid length-scale ${ }^{83}$ is beeing investigated within LAVA.

In summary, both approaches considered (LBM-LES and ZDES Mode 3) make predictions that are in excellent agreement with measurements and other simulations from literature. There is a significant difference in resolution requirements between the two approaches due to the underlying grid-paradigm used. The Cartesian method requires significantly more grid points to resolve the devoloping turbulent boundary layer on account of its isotropic nature. The use of mesh refinement and higher order boundary representation helps to alleviate this limitation to a certain extent. Yet, for problems where resolving the boundary layer development over the underlying geometry is essential for accurate predictions of the flow physics, Cartesian methods are disproportionally expensive. On the other hand, for flows where the solution quality is not dominated by the resolution of the turbulent boundary layer, the LBM method on Cartesian meshes has demonstrated a significant performance benefit without any compromise in accuracy or robustness whatsoever (see Barad et al. ${ }^{45}$ ).

Finally, the efficiency of the two methods presented in this study is evaluated based on the number of CPU hours required for one convective time unit. The simulations were performed on the Pleiades cluster at NASA Ames Research Center using Intel Xeon E5-2680v4 processors. A total of 240 CPUh were necessary for the ZDES Mode 3 approach compared to 614 CPUh using the LBM-LES method. The superior computational efficiency of the body-fitted curvilinear method relative to the Cartesian immersed boundary LBM (by a factor of 2.5) is due to the substantially larger timestep size of $\Delta t=1.8 \times 10^{-5}$ used by the former relative to $\Delta t=4.6 \times 10^{-7}$ used by the latter (by a factor of 39). As reported in Duda et al. ${ }^{4}$ comparable simulations using a hybrid RANS/LES paradigm within LBM are approximately $13 \%$ faster for similar solution accuracy ( $540 \mathrm{CPUh}$ per convective time unit, $\Delta t=6.868 \times 10^{-7}$ ). The hybrid RANS/LES approach has the advantage of less stringent resolution requirements. ${ }^{4}$ Therefore a hybrid RANS/LES approach is currently under development within LAVA-LBM. We remind the reader that the conclusions drawn here regarding the relative computational efficiency of the two approaches for comparable accuracy is specific to the particular test case under consideration. Furthermore, in practice the decision to favor a Cartesian approach over a body-fitted approach almost always hinges on the complexity of the underlying geometry and the significantly larger manual effort required to generate a body-fitted mesh.

Table 2: Separation and reattachment locations and corresponding separation bubble size from different LAVA simulations, data from literature and experiments from Greenblatt et al. ${ }^{1}$ Measurements for DDES are taken at second reatachment location ignoring bifurcated flow region. Separation bubble size error was calculated based on Eqn 3 .

\begin{tabular}{lcccc}
\hline Case & $\begin{array}{c}\text { Separation Location } \\
(\mathrm{x} / \mathrm{c})\end{array}$ & $\begin{array}{c}\text { Reattachment Location } \\
(\mathrm{x} / \mathrm{c})\end{array}$ & $\begin{array}{c}\text { Bubble length } \\
(\Delta \mathrm{x} / \mathrm{c})\end{array}$ & $\begin{array}{c}\text { Error } \\
(\%)\end{array}$ \\
\hline Greenblatt et al. & $0.665 \pm 0.005$ & $1.10 \pm 0.005$ & 0.435 & \\
LAVA RANS fine 2D & 0.661 & 1.263 & 0.602 & 38.3 \\
LAVA DDES & 0.659 & 1.343 & 0.684 & 57.2 \\
LAVA DDES + SEM & 0.659 & 1.230 & 0.571 & 31.2 \\
LAVA ZDES Mode 3 & 0.658 & 1.115 & 0.457 & 5.1 \\
LAVA LBM-LES & 0.662 & 1.128 & 0.466 & 7.1 \\
Uzun et al. & 0.660 & 1.090 & 0.430 & -1.1 \\
PowerFlow fine LBM-VLES & 0.673 & 1.153 & 0.480 & 10.3 \\
\hline
\end{tabular}



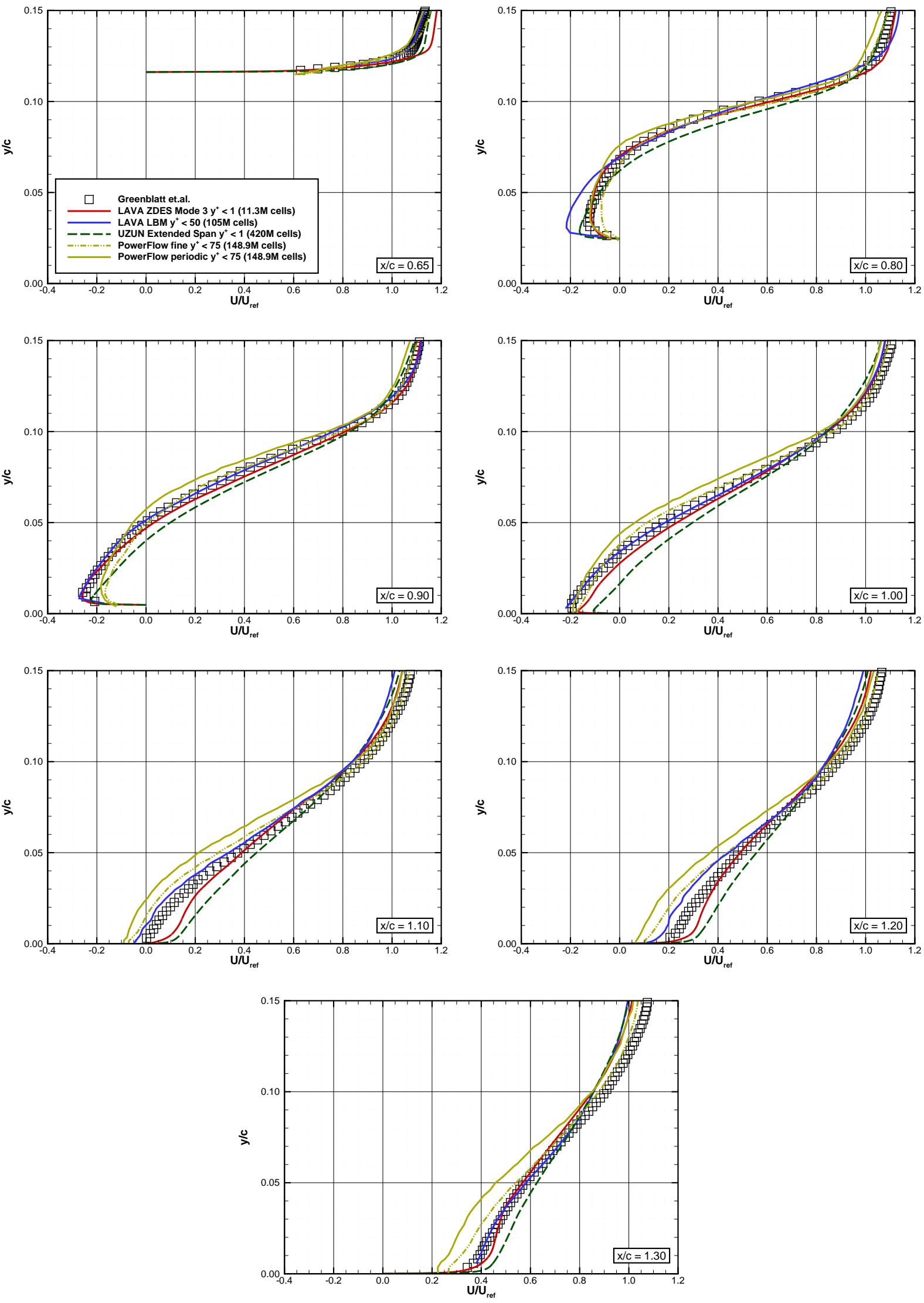

Figure 23: Time averaged u-velocity profiles extracted at seven different locations downstream of the separation location. WMLES data from Uzun and Malik ${ }^{3}$ and VLES-LBM data from Duda and Fares ${ }^{4}$ digitized from referenced papers. 

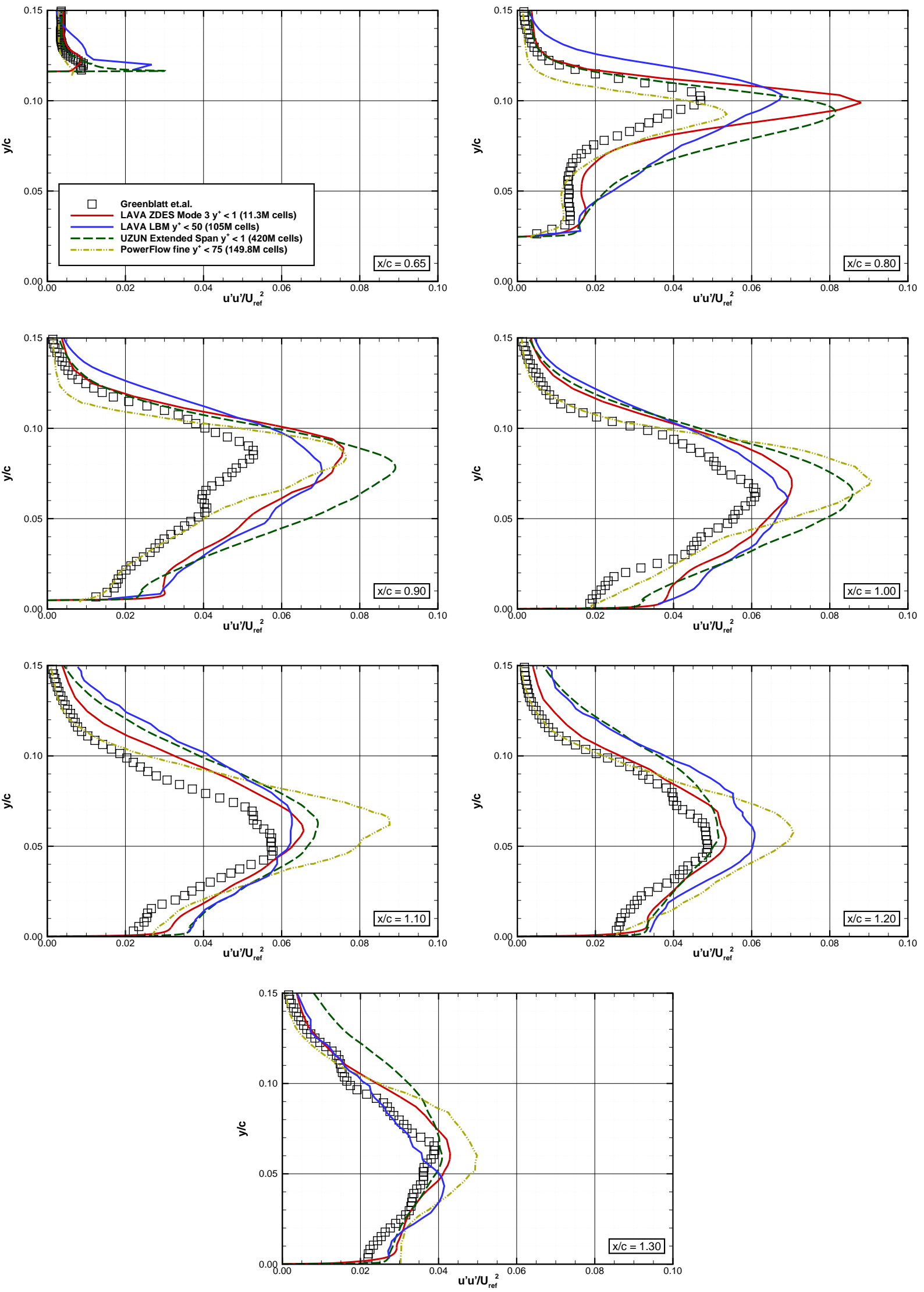

Figure 24: RMS $u^{\prime} u^{\prime}$ u-velocity profiles extracted at seven different locations downstream of the separation location. WMLES data from Uzun and Malik ${ }^{3}$ and VLES-LBM data from Duda and Fares ${ }^{4}$ digitized from referenced papers. 

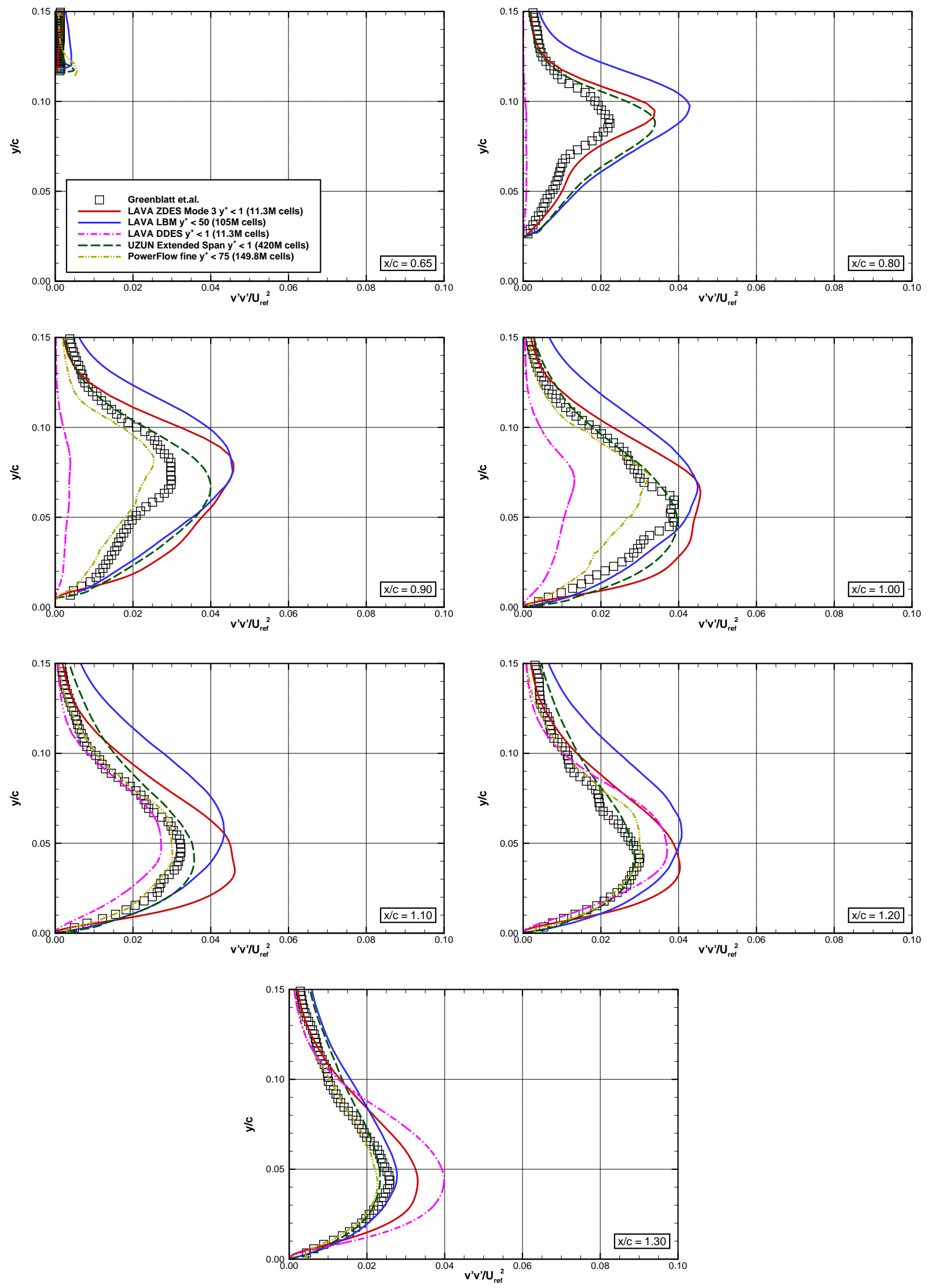

Figure 25: RMS $v^{\prime} v^{\prime}$ profiles extracted at seven different locations downstream of the separation location. WMLES data from Uzun and Malik ${ }^{3}$ and VLES-LBM data from Duda and Fares ${ }^{4}$ digitized from referenced papers. 

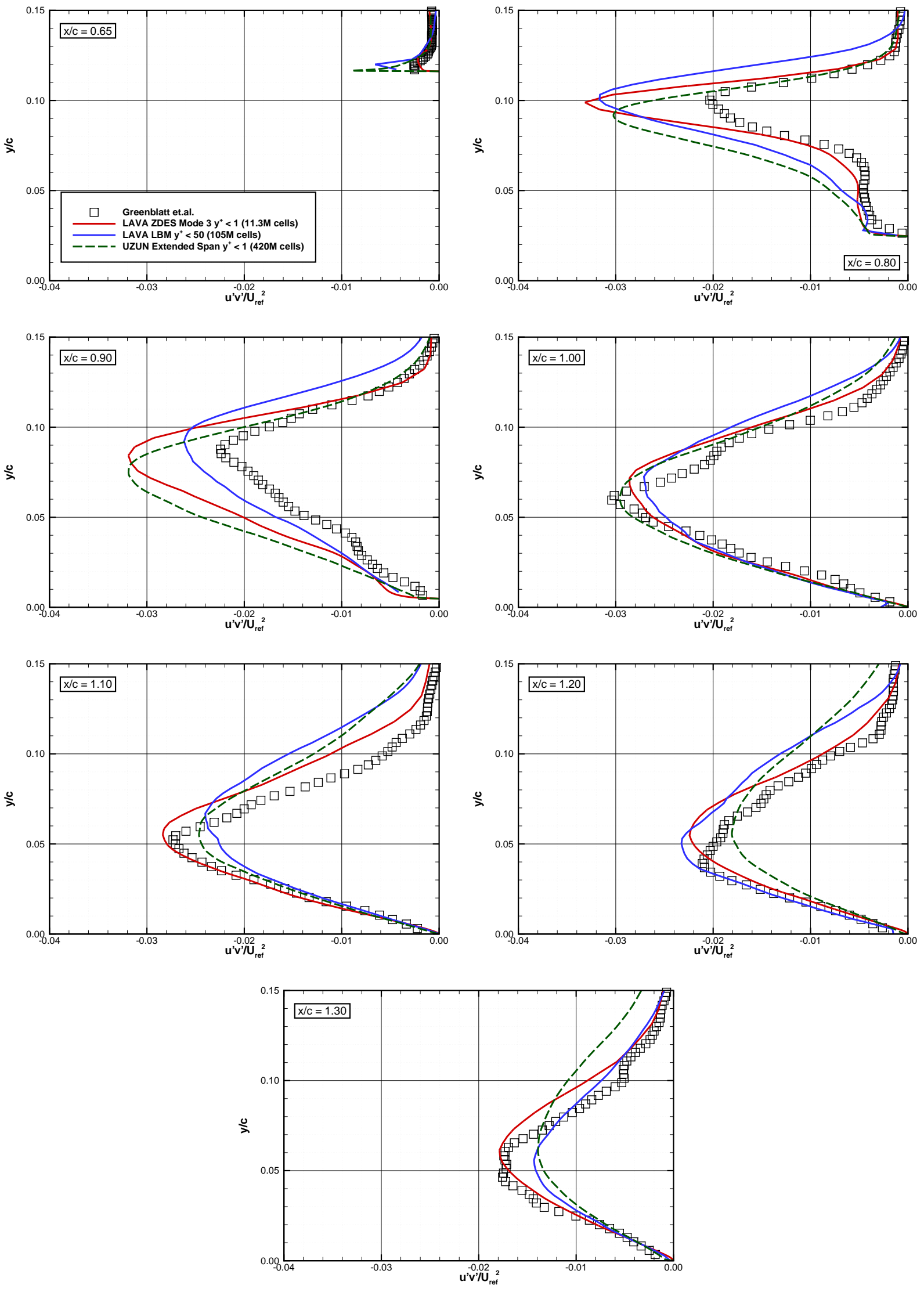

Figure 26: RMS $u^{\prime} v^{\prime}$ profiles extracted at seven different locations downstream of the separation location. WMLES data from Uzun and Malik ${ }^{3}$ and VLES-LBM data from Duda and Fares ${ }^{4}$ digitized from referenced papers. 


\section{Summary}

RANS, DDES, ZDES Mode 3 as well as LBM-LES predictions have been presented and compared to experimental data as well as other results from literature, ${ }^{4,8}$ for NASA's wall mounted hump test case. Both Lattice Boltzmann using Cartesian grids and hybrid RANS/LES using structured overlapping grids were able to achieve excellent agreement with experimental data. The effects of grid resolution were studied for RANS simulations and the well known deficiencies of state-of-the-art turbulence models in predicting separation and reattachment were demonstrated. RANS models generally underpredict the Reynolds stresses in the separated shear layer, where the flow is far away from equilibrium, resulting in delayed reattachment. The error in predicting the separation bubble size has been found to be consistent with previous RANS simulations reported at around $38 \%$. Similar, if not even worse behavior has been observed with SADDES even when using an improved length scale. ${ }^{16}$ The insufficient production of turbulent structures leeward of the separation location causes a delayed reattachment and thus creates an even larger separation bubble relative to RANS. Recently, improvements aimed at enhancing the production of turbulence using the DDES approach in conjunction with a shear layer adapted definition of the subgrid length-scale have shown promising results. ${ }^{83}$ Those modifications to the SA-DDES model together with the Improved DDES (IDDES) will be considered in future work. ZDES Mode 3 where RANS acts as a wall-model for the outer LES simulation showed good agreement with velocity and Reynolds stress profiles as well as skin friction and pressure coefficient distribution measured by Greenblatt et al. ${ }^{1}$ No delay in the development of realistic 3D turbulence in the shear layer that originates from the separation point has been observed in the ZDES Mode 3 simulations. Sensitivity of skin friction distribution and resolved Reynolds stress profiles to the location of the RANS/LES interface for a fixed mesh has been documented. A timestep sensitivity study for ZDES was performed and only minor differences were found. The LBM-LES method within LAVA gave excellent results when comparing velocity profiles and Reynolds stress profiles. Blockage effects due to side walls in the experiment need to be investigated further. In order to lower the cell count necessary in our LBM-LES simulation, the development of a hybrid RANS/LES model within the LBM framework is currently being pursued.

Both the ZDES Mode 3 as well as LBM-LES simulations met the technical challenge (TC) created by NASA's Revolutionary Computational Aerosciences (RCA) program to reduce the predictive error of canonical separated flows by $40 \%$. An improvement of more than $90 \%$ was demonstrated using both approaches presented in this paper. The superior computational efficiency of the body-fitted curvilinear method relative to the Cartesian immersed boundary LBM for a given accuracy was demonstrated. Due to the isotropic nature of the computational cells within the LBM solver and the explicit nature of time advancement, the resolution requirements were significantly higher, thus negating the performance benefits of the latter relative to the former. This particular problem is a well-known limitation of the Cartesian paradigm where resolving the boundary layer development over the underlying geometry is essential for accurate prediction of the flow physics. Despite the increased resolution requirements, Cartesian methods especially when combined with the efficiency of Lattice Boltzmann method have the critical advantage that manual volumetric mesh generation efforts are completely eliminated.

It is worth pointing out that the RCA technical challenge focuses on canonical configurations with minimal geometric complexity. Nearly all geometries with a few exceptions (eg. the Common Research Model, Wingbody-juncture) are relatively straight forward to mesh even when using a structured mesh paradigm. The meshing efforts increase exponentially when the underlying geometry is extremely complex and can often require hundreds of hours of manual labor. Cartesian methods on the other hand completely eliminate this process resulting in much faster turnaround times. For this reason we consider multiple grid paradigms and numerical methods absolutely necessary for future CFD work. ${ }^{9}$

\section{Acknowledgements}

This work was funded by the NASA Transformational Tools and Technologies Project (TTT) of the Transformative Aeronautics Concepts Program under the Aeronautics Research Mission Directorate (ARMD). Computer time has been provided by the NASA Advanced Supercomputing (NAS) facility at NASA Ames Research Center. We would like to thank our colleauges from NASA's Computational Aerosciences Branch for many useful discussions. 


\section{References}

${ }^{1}$ Greenblatt, D., Paschal, K., Yao, C.-S., Harris, J., Schaeffler, N., and Washburn, A., "Experimental Investigation of Separation Control Part 1: Baseline and Steady Suction," AIAA Journal, Vol. 44, No. 12, 2006, pp. 2820-2830.

${ }^{2}$ Iyer, P. and Malik, M., "Wall-Modeled Large-Eddy Simulation of a Separated Flow Over the NASA Wall-Mounted Hump," 56th AIAA Fluid Dynamics Conference, June 13-17 2016, AIAA-2016-3186.

${ }^{3}$ Uzun, A. and Malik, M., "Wall-Resolved Large-Eddy Simulation of Flow Separation Over NASA Wall-Mounted Hump," 55th AIAA Aerospace Sciences Meeting, January 9-13 2017, AIAA-2017-0538.

${ }^{4}$ Duda, B. and Fares, E., "Application of a Lattice-Boltzmann Method to the Separated Flow Behind the NASA Hump," 54th AIAA Aerospace Sciences Meeting, January 4-8 2016, AIAA-2016-1836.

${ }^{5}$ Spalart, S. R., Jou, W.-H., Strelets, M., and Allmaras, S. R., "Comments on the feasibility of LES for wings, and on a hybrid RANS/LES approach," First AFOSR international conference on DNS/LES, Ruston, Louisiana, August 1997, Greyden Press.

${ }^{6}$ Seifert, A. and Pack, L., "Active flow separation controll on wall-mounted hump at high Reynolds numbers." AIAA Journal, Vol. 40, No. 7, 2002, pp. 1363-1372.

${ }^{7}$ You, D., Wang, M., and Moin, P., "Large-Eddy Simulation of Flow over a Wall-Mounted Hump with Separation Controll," AIAA Journal, Vol. 44, November 2006.

${ }^{8}$ Uzun, A. and Malik, M., "Large-Eddy Simulation of Flow over a Wall-Mounted Hump with Separation and Reattachment," AIAA Journal, Vol. 56, February 22018.

${ }^{9}$ Kiris, C., Housman, J., Barad, M., Brehm, C., Sozer, E., and Moini-Yekta, S., "Computational Framework for Launch, Ascent, and Vehicle Aerodynamics (LAVA)," Aerospace Science and Technology, Vol. 55, August 2016, pp. 189-219.

${ }^{10}$ Steger, J. and Benek, J., "On the Use of Composite Grid Schemes in Computational Aerodynamics," Technical Memorandum 88372, NASA, 1986.

${ }^{11}$ Chan, W., "Developments in Strategies and Software Tools for Overset Structured Grid Generation and Connectivity," 20th AIAA Computational Fluid Dynamics Conference, Honolulu, Hawaii, June 2011, AIAA-2011-3051.

${ }^{12}$ Vinokur, M., "Conservation Equations of Gasdynamics in Curvilinear Coordinate Systems," Journal of Computational Physics, Vol. 14, 1974, pp. 105-125.

${ }^{13}$ Spalart, S. and Allmaras, S., "A One-Equation Turbulence Model for Aerodynamic Flows," 30th Aerospace Sciences Meeting and Exhibit, Reno, NV, January 1992, AIAA-92-0439.

${ }^{14}$ Brehm, C., Housman, J., Kiris, C., and Hutcheson, F., "Noise Characteristics of a Four-Jet Impingement Device Inside a Broadband Engine Noise Simulator," 21st AIAA/CEAS Aeroacoustics Conference, Dallas, Texas, June 2015, AIAA-2015-2211.

${ }^{15}$ Housman, J. and Kiris, C., "Structured Overlapping Grid Simulations of Contra-Rotating Open Rotor Noise," 54 th AIAA Aerospace Sciences Meeting, January 4-8 2016, AIAA-2016-0814.

${ }^{16}$ Housman, J. and Kiris, C., "Slat Noise Predictions using Higher-Order Finite-Difference Methods on Overset Grids," 22nd AIAA/CEAS Aeroacoustic Conference, Lyon, France, May 30-31 2016, AIAA-2016-2963.

${ }^{17} \mathrm{Brehm}$, C., Housman, J., and Kiris, C., "Noise Generation Mechanisms for a supersonic jet impinging on an inclined plate," Journal of Fluid Mechanics, Vol. 797, 2016, pp. 802-850.

${ }^{18}$ Brehm, C., Barad, M., Housman, J., and Kiris, C., "A Comparison of Higher-Order Finite-Difference Shock Capturing Schemes," Computers 83 Fluids, Vol. 122, November 2015, pp. 184-208.

${ }^{19}$ Shu, C.-W., "High Order Weighted Essentially Nonoscillatory Schemes for Convection Dominated Problems," SIAM Review, Vol. 51, No. 1, 2009, pp. 92-126.

${ }^{20}$ Deng, X. and H., Z., "Developing High-Order Weighted Compact Nonlinear Schemes," Journal of Computational Physics, Vol. 165, 2000, pp. 22-44.

${ }^{21}$ Vinokur, M. and Yee, H., "Extension of Efficient Low Dissipation High Order Schemes for 3-D Curvilinear Moving Grids," Frontiers of Computational Fluid Dynamics, World Scientific, 2002, pp. 129-163, edited by D. A. Caughey and M. M. Hafez.

${ }^{22}$ Deng, X., Mao, M., Tu, G., Liu, H., and Zhang, H., "Geometric Conservation Law and Applications to High-Order Finite Difference Schemes with Stationary Grids," Journal of Computational Physics, Vol. 230, 2011, pp. 1100-1115.

${ }^{23}$ Housman, J., Kiris, C., and Hafez, M., "Preconditioned methods for simulations of low speed compressible flows," Computers 8 Fluids, Vol. 38, No. 7, August 2009, pp. 1411-1423.

${ }^{24}$ Housman, J., Kiris, C., and Hafez, M., "Time-Derivative Preconditioning Methods for Multicomponent Flows - Part I: Riemann Problems," Journal of Applied Mechanics, Vol. 76, No. 2, February 2009.

${ }^{25}$ Housman, J., Kiris, C., and Hafez, M., "Time-Derivative Preconditioning Methods for Multicomponent Flows - Part II: Two-Dimensional Applications," Journal of Applied Mechanics, Vol. 76, No. 3, March 2009.

${ }^{26}$ Deng, X., Mao, M., Jiang, Y., and Liu, H., "New High-Order Hybrid Cell-Edge and Cell-Node Weighted Compact Nonlinear Schemes," 20th AIAA Computational Fluid Dynamics Conference, Honolulu, Hawaii, June 2011, AIAA-2011-3857.

${ }^{27}$ Nonomura, T. and Fujii, K., "Robust Explicit Formulation of Weighted Compact Nonlinear Scheme," Computers \& Fluids, Vol. 85, 2013, pp. 8-18.

${ }^{28}$ Strelets, M., "Detached eddy simulation of massively separated flows," 39th Aerospace Sciences Meeting and Exhibit, Reno, Nevada, January 2001, AIAA-2001-0879.

${ }^{29}$ Spalart, P., Deck, S., Shur, M., Squires, K., Strelets, M., and Travin, A., "A New Version of Detached-Eddy Simulation, Resistant to Ambiguous Grid Densities," Theoretical and Computational Fluid Dynamics, Vol. 20, May 2006, pp. 181-195.

${ }^{30}$ Shur, M., Spalart, P. R., Strelets, M., and Travin, A., "A Hybrid RANS-LES Approach with Delayed-DES and WallModelled LES Capabilities," International Journal of Heat and Fluid Flow, Vol. 29, 2008, pp. 1638-1649.

${ }^{31}$ Spalart, P. R., "Detached-Eddy Simulation," Annual Review Fluid Mechanics, Vol. 41, 2009, pp. 181-202.

${ }^{32}$ Caruelle, B. and Ducros, F., "Detached-Eddy Simulations of Attached and Detached Boundary Layers," International Journal of Computational Fluid Dynamics, Vol. 17, 2003, pp. 433-451. 
${ }^{33}$ Ashton, N., West, A., and Mendonca, F., "Flow Dynamics Past a 30P30N Three-Element Airfoil Using Improved Delayed Detached-Eddy Simulation," AIAA Journal, Vol. 54, No. 11, 2016, pp. 3657-3667.

${ }^{34}$ Chauvet, N., Deck, S., and Jacquin, L., "Zonal Detached Eddy Simulation of a Controlled Propulsive Jet," AIAA Journal, Vol. 45, No. 10, 2007, pp. 2458-2473.

${ }^{35}$ Deck, S., "Recent Improvements in the Zonal Detached-Eddy Simulation (ZDES) Formulation," Theoretical and Computational Fluid Dynamics, Vol. 26, 2012, pp. 523-550.

${ }^{36}$ Shur, M. e., "An Enhanced Version of DES with Rapid Transition from RANS to LES in Separated Flows," Flow, Turbulence and Combustion, Vol. 95, No. 4, December 2015, pp. 709-737.

${ }^{37}$ Deck, S., "Numerical simulation of transonic buffed over a supercritical airfoil," AIAA Journal, Vol. 43, 2005, pp. 15561566.

${ }^{38}$ Deck, S., "Zonal-detached eddy simulation of the flow around a high-lift configuration," AIAA Journal, Vol. 43, 2005, pp. $2372-2384$.

${ }^{39}$ Eastwood, S., Xia, H., and Tucker, P., "Large-Eddy Simulation of Complex Geometry Jets," Journal of Propulsion and Power, Vol. 28, No. 2, March-April 2012, pp. 235-245.

${ }^{40}$ Zhu, H., Fu, S., Shi, L., and Wang, Z., "A Hybrid RANS-Implicit LES Approach for the High-Order FR/CPR Method," 54th AIAA Aerospace Sciences Meeting, San Diego, Ca., January 2016, AIAA-2016-1599.

${ }^{41}$ Deck, S., Weiss, P., Pamies, M., and Garnier, E., "Zonal Detached Eddy Simulation of s spatially developing flat plate turbulent boundary layer," Computers and Fluids, Vol. 48, 2011, pp. 1-15.

${ }^{42}$ Deck, S., Renard, N., Laraufie, R., and Sagaut, P., "Zonal detached eddy Simulation (ZDES) of a spatially developing flat plate turbulent boundary layer over the Reynolds number range $3150 \leq R e_{\theta} \leq 14000$," Physics of Fluids, Vol. $26,2014$.

${ }^{43}$ Renard, N. and Deck, S., "Improvements in Zonal Detached Eddy Simulation for Wall Modeled Large Eddy Simulation," AIAA Journal, Vol. 53, No. 11, 2015.

${ }^{44}$ Chen, S. and Doolen, G. D., "Lattice Boltzmann method for fluid flows," Annual review of fluid mechanics, Vol. 30, No. 1, 1998, pp. 329-364.

${ }^{45}$ Barad, M., Kocheemoolayil, J., and Kiris, C., "Lattice Boltzmann and Navier-Stokes Cartesian CFD Approaches for Airframe Noise Predictions," AIAA Aviation Forum, Vol. 23, 2017.

${ }^{46}$ Barad, M., Kocheemoolayil, J., Stich, G., and Kiris, C., "Progress of Lattice Boltzmann Development in the LAVA Framework," Tenth International Conference on Computational Fluid Dynamics, Vol. 10, 2018.

${ }^{47}$ Chapman, S. and Cowling, T. G., The mathematical theory of non-uniform gases: an account of the kinetic theory of viscosity, thermal conduction and diffusion in gases, Cambridge university press, 1970.

${ }^{48}$ Bhatnagar, P. L., Gross, E. P., and Krook, M., "A Model for Collision Processes in Gases. I. Small Amplitude Processes in Charged and Neutral One-Component Systems," Physical Review E, Vol. 94, 1954, pp. 511-525.

${ }^{49} \mathrm{Li}$, Y., Shock, R., Zhang, R., and Chen, H., "Numerical study of flow past an impulsively started cylinder by the lattice-Boltzmann method," Journal of Fluid Mechanics, Vol. 519, 2004, pp. 273-300.

${ }^{50}$ S. Ansumali, I. V. K. and Öttinger, H. C., "Minimal entropic kinetic models for hydrodynamics," Europhysics Letters, Vol. 63, No. 6, 2003, pp. 798-804.

${ }^{51}$ Lallemand, P. and Luo, L.-S., "Theory of the lattice Boltzmann method: Dispersion, dissipation, isotropy, Galilean invariance, and stability," Physical Review E, Vol. 61, Jun 2000, pp. 6546-6562.

${ }^{52}$ Bosch, F., Chikatamarla, S. S., and Karlin, I. V., "Entropic multirelaxation lattice Boltzmann models for turbulent flows," Physical Review E, Vol. 92, 2015, pp. 043309.

${ }^{53}$ Latt, J. and Chopard, B., "Lattice Boltzmann method with regularized pre-collision distribution functions," Mathematics and Computers in Simulation, Vol. 72, No. 2-6, 2006, pp. $165-168$.

${ }^{54}$ Smagorisnky, J., "General circulation experiments with the primitive equations," Monthly Weather Review, Vol. 91, No. 3, 1963, pp. 99-164.

${ }^{55}$ M'hamed Bouzidi, M. F. and Lallemand, P., "Momentum transfer of a Boltzmann-lattice fluid with boundaries," Physics of Fluids, Vol. 13, No. 11, 2001, pp. 3452-3459.

${ }^{56}$ Larsson, J., Kawai, S., Bodart, J., and Bermejo-Moreno, I., "Large Eddy Simulation with Modeled Wall-Stress: Recent Progress and Future Directions," J-STAGE, 2015.

${ }^{57}$ Bose, S. and Park, G., "Wall-Modeled Large-Eddy Simulation for Complex Turbulent Flows," Annual Review of Fluid Mechanics, Vol. 50, 2018, pp. 535-561.

${ }^{58}$ Malaspinas, O.Sagaut, P., "Wall model for large-eddy simulation based on the lattice Boltzmann method," Physics of Fluids, Vol. 24, 2012.

${ }^{59}$ Berger, M. J. and Oliger, J. E., "Adaptive mesh refinement for hyperbolic partial differential equations," Tech. rep., Stanford University, Stanford, CA, USA, 1983.

${ }^{60}$ Berger, M. J. and Colella, P., "Local Adaptive Mesh Refinement for Shock Hydrodynamics," Journal of Computational Physics, Vol. 82, No. 1, May 1989, pp. 64-84.

${ }^{61}$ Almgren, A. S., Bell, J. B., Colella, P., Howell, L. H., and Welcome, M. L., "A Conservative Adaptive Projection Method for the Variable Density Incompressible Navier-Stokes Equations," J. Comp. Phys., Vol. 142, 1998, pp. 1-46.

${ }^{62}$ Barad, M. F. and Colella, P., "A Fourth-Order Accurate Local Refinement Method for Poisson's Equation," J. Comp. Phys., Vol. 209, No. 1, October 2005, pp. 1-18.

${ }^{63}$ Barad, M. F., Colella, P., and Schladow, S. G., "An Adaptive Cut-Cell Method for Environmental Fluid Mechanics," Int. J. Numer. Meth. Fluids, Vol. 60, No. 5, 2009, pp. 473-514.

${ }^{64}$ Zhang, Q., Johansen, H., and Colella, P., "A Fourth-Order Accurate Finite-Volume Method with Structured Adaptive Mesh Refinement for Solving the Advection-Diffusion Equation," SIAM Journal on Scientific Computing, Vol. 34, No. 2, 2012, pp. 179-201. 
${ }^{65}$ Colella, P., Graves, D. T., Ligocki, T. J., Martin, D. F., Modiano, D., Serafini, D. B., and Straalen, B. V., "Chombo Software Package for AMR Applications - Design Document," unpublished.

${ }^{66}$ Martin, D. F., Colella, P., and Graves, D., "A Cell-Centered Adaptive Projection Method for the Incompressible NavierStokes Equations in Three Dimensions," Journal of Computational Physics, Vol. 227, 2008, pp. 1863-1886.

${ }^{67}$ Chen, H., Filippova, O., Hoch, J., Molvig, K., Shock, R., Teixeira, C., and Zhang, R., "Grid refinement in lattice Boltzmann methods based on volumetric formulation," Physica A: Statistical Mechanics and its Applications, Vol. 362, No. 1, 2006, pp. 158-167.

${ }^{68}$ Rohde, M., Kandhai, D., Derksen, J., and Van den Akker, H., "A generic, mass conservative local grid refinement technique for lattice-Boltzmann schemes," International journal for numerical methods in fluids, Vol. 51, No. 4, 2006, pp. 439468.

${ }^{69}$ Jarrin, N., Benhamadouche, S., Laurence, D., and Prosser, R., "A Synthetic-Eddy-Method for Generating Inflow Conditions for Large-Eddy Simulations," International Journal of Heat and Fluid Flow, Vol. 27, 2006, pp. 585-593.

${ }^{70}$ Jarrin, N., Prosser, R., Uribe, J.-C., Benhamadouche, S., and Laurence, D., "Reconstruction of Turbulent Fluctuations for Hybrid RANS/LES simulations using a Synthetic-Eddy Method," International Journal of Heat and Fluid Flow, Vol. 30, 2009 , pp. 435-442.

${ }^{71}$ Shur, M., Spalart, P., Strelets, M., and Travin, A., "Synthetic Turbulence Generators for RANS-LES Interfaces in Zonal Simulations of Aerodynamic and Aeroacoustic Problems," Flow Turbulence and Combustion, Vol. 93, 2014, pp. 63-92.

${ }^{72}$ Rumsey, C., "Turbulence Modeling Resource," https://www.turbmodles.larc.nasa.gov, Online; accessed 2018-04-30.

${ }^{73}$ Iyer, P., Park, G., and Malik, M., "Wall-Modeled Large-Eddy Simulation of Transonic Flow over an Axisymmetric Bump with Shock-Induced Separation," 23rd AIAA Computational Fluid Dynamics Conference, June 5-9 2017, AIAA-2017-3953.

${ }^{74}$ Rumsey, C., "Turbulence Modeling Resource - 2D Wall-Mounted hump," https://www.turbmodles.larc.nasa.gov/ nasahump_grids.html, Online; accessed 2018-04-30.

${ }^{75}$ Slotnick, J. e. a., "CFD Vision 2030 Study: A Path to Revolutionary Computational Aerosciences," NASA Technical Report, 2014, NASA/CR-2014-218178.

${ }^{76}$ Rumsey, C. and Jeyapaul, E., "Pressure-strain and near-wall modeling for two-dimensional separatef flows," Center for Turbulence Research, 2012.

${ }^{77}$ Eisfeld, B. Rumsey, C. and Togiti, V., "Second-Moment RANS Model Verification and Validation using the Turbulence Modeling Resources Website (invited)," AIAA Aviation Forum, 2015, AIAA-2015-2924.

${ }^{78}$ Lardeau, S. and Billard, F., "Development of an elliptic-blending lag model for industrial applications," AIAA Scitech Forum, 2016, AIAA 2016-1600.

${ }^{79}$ Park, G., "Wall-modeled LES in unstructured grids: Application to the NASA wall-mounted Hump," 54th AIAA Aerospace Sciences Meeting, 2016, AIAA-2016-1560.

${ }^{80}$ Garbaruk, A., Guseva, E., Shur, L., Strelets, M., and Travin, A., "2D Wall-Mounted Hump," Notes on Numerical Fluid Mechanics and Multidisciplinary Design, 2018.

${ }^{81}$ Probst, A., Schwamborn, D., Garbaruk, A., Guseva, E., Shur, M., M., S., and Travin, A., "Evaluation of grey area mitigation tools within zonal and non-zonal RANS-LES approaches in flows with pressure induced separation," International Journal of Heat and Fluid Flow, 2017, pp. 1-11.

${ }^{82}$ Housman, J., Stich, G., and Kiris, C., "Jet Noise Prediction using Hybrid RANS/LES with Structured Overset Grids," 23rd AIAA/CEAS Aeroacoustic Conference, Denver, Colorado, 2017, AIAA-2017-3213.

${ }^{83}$ Guseva, E., Garbaruk, A., and Strelets, M., "Assessment of Delayed DES and Improved Delayed DES Combined with a Shear-Layer-Adapted Subgrid Length-Scale in Separated Flows," Flow Turbulence Combustion, 2017. 